\title{
VARIATIONAL ANALYSIS OF DIRECTIONAL MINIMAL TIME FUNCTIONS AND APPLICATIONS TO LOCATION PROBLEMS
}

\author{
Nguyen Mau Nam ${ }^{1}$ and Constantin Zălinescu ${ }^{2}$
}

\begin{abstract}
This paper is devoted to the study of directional minimal time functions that specify the minimal time for a vector to reach an object following its given direction. We provide a careful analysis of general and generalized differentiation properties of this class of functions. The analysis allows us to study a new model of facility location that involves sets. This is a continuation of our effort in applying variational analysis to facility location problems.
\end{abstract}

Key words. Directional minimal time functions, scalarization functions, generalized differentiation, facility location problems.

\section{Introduction and Preliminaries}

Let $X$ be a real normed linear space. Given a vector $v \in X, v \neq 0$, and a nonempty closed set $\Omega \subseteq X$, the directional minimal time function with direction $v$ and target set $\Omega$ is defined by

$$
T_{v}(x ; \Omega):=\inf \{t \geq 0 \mid x+t v \in \Omega\} .
$$

This class of functions is similar to the class of scalarization functions that has been used to study vector optimization problems:

$$
\varphi_{v}(x ; \Omega):=\inf \{t \in \mathbb{R} \mid x+t v \in \Omega\}
$$

see $[6,11]$ and the references therein. We will see later on that if $v \in \Omega_{\infty}$, then

$$
T_{v}(x ; \Omega)=\max \left\{\varphi_{v}(x ; \Omega), 0\right\} \quad \forall x \in X .
$$

Lipschitz properties and a formula for computing subdifferentials in the sense of convex analysis of scalarization functions were studied in [11], but generalized differentiation properties involving nonconvex structures have not been considered in the literature. Notice that the directional minimal time function (1) is a particular case of general minimal time functions considered, e.g., in $[5,8]$ and the references therein. However, the specific structure of the function makes it distinct from the general case.

In this paper, we mainly study generalized differentiation properties the directional minimal time function (1) and the scalarization function (2), as well as applications to facility location problems. The location model that motivates our study is a generalized version of the celebrated Fermat-Torricelli problem: given a finite number of nonempty

\footnotetext{
${ }^{1}$ Fariborz Maseeh Department of Mathematics and Statistics, Portland State University, Portland, OR 97202, United States (email: mau.nam.nguyen@pdx.edu). The research of Nguyen Mau Nam was partially supported by the Simons Foundation under grant \#208785.

${ }^{2}$ University Al.I. Cuza Iaşi, Faculty of Mathematics, 700506 Iaşi, Romania (email: zalinesc@uaic.ro).
} 
closed target sets $\Omega_{i}$ for $i=1, \ldots, n$ and $n$ nonzero vectors $v_{i}$ for $i=1, \ldots, n$, along with a nonempty closed constraint set $\Omega_{0}$, find a point $\bar{x} \in \Omega_{0}$ to place the initial points of the vectors such that the total times for the vectors to reach the target sets is minimal. This problem can be modeled as the following optimization problem:

$$
\operatorname{minimize} \sum_{i=1}^{n} T_{v_{i}}\left(x ; \Omega_{i}\right) \text { subject to } x \in \Omega_{0} .
$$

The location model of this type seems to be very interesting, but it has not been considered in the literature even in the convex case. Since the functions involved in the problem are not differentiable in general, our approach involves developing new tools of modern variational analysis for these functions and apply them to solve the problem. Let us recall basic definitions and properties of variational analysis that will play an important role in the sequel.

Let $\Omega \subseteq X$ and let $\bar{x} \in \Omega$. A vector $x^{*} \in X^{*}$ is called a Fréchet normal to $\Omega$ at $\bar{x}$ if

$$
\left\langle x^{*}, x-\bar{x}\right\rangle \leq \mathrm{o}(\|x-\bar{x}\|) \text { for } x \in \Omega \text {. }
$$

The set of all Fréchet normals to $\Omega$ at $\bar{x}$ is called the Fréchet normal cone to $\Omega$ at $\bar{x}$, denoted by $\widehat{N}(\bar{x} ; \Omega)$.

A vector $x^{*} \in X^{*}$ is called a Mordukhovich/limiting normal to $\Omega$ at $\bar{x}$ if there are sequences $x_{k} \stackrel{\Omega}{\rightarrow} \bar{x}$ and $x_{k}^{*} \stackrel{w^{*}}{\rightarrow} x^{*}$ with $x_{k}^{*} \in \widehat{N}\left(x_{k} ; \Omega\right)$ for every $k$. The set of all Mordukhovich normals to $\Omega$ at $\bar{x}$ is called the Mordukhovich/limiting normal cone to the set at this point. In this definition, the notation $x_{k} \stackrel{\Omega}{\rightarrow} \bar{x}$ means that $x_{k} \rightarrow \bar{x}$ and $x_{k} \in \Omega$ for every $k$.

Let $\psi: X \rightarrow(-\infty, \infty]$ be an extended real-valued function and let $\bar{x}$ be an element of the domain of the function dom $\psi:=\{x \in X \mid \psi(x)<\infty\}$. The Fréchet subdifferential of $\psi$ at $\bar{x}$ is defined by

$$
\widehat{\partial} \psi(\bar{x}):=\left\{x^{*} \in X^{*} \mid\left\langle x^{*}, x-\bar{x}\right\rangle \leq \psi(x)-\psi(\bar{x})+\mathrm{o}(\|x-\bar{x}\|)\right\} \text { for } x \in X .
$$

The Mordukhovich/limiting subdifferential of $\psi$ at $\bar{x}$, denoted by $\partial \psi(\bar{x})$, is the set of all vectors $x^{*} \in X^{*}$ such that there exist sequences $x_{k} \stackrel{\psi}{\rightarrow} \bar{x}$, and $x_{k}^{*} \in \widehat{\partial} \psi\left(x_{k}\right)$ with $x_{k}^{*} \stackrel{w^{*}}{\longrightarrow} x^{*}$, where $x_{k} \stackrel{\psi}{\rightarrow} \bar{x}$ means that $x_{k} \rightarrow \bar{x}$ and $\psi\left(x_{k}\right) \rightarrow \psi(\bar{x})$.

In the case where the set $\Omega$ is convex or the function $\psi$ is convex, the corresponding Fréchet normal cone and subdifferential structures reduce respectively to those in the sense of convex analysis. If $X$ is a Banach space, then the same holds for the limiting structures.

The singular subdifferential of $\psi$ at $\bar{x}$, denoted by $\partial^{\infty} \psi(\bar{x})$, is the set of all vectors $x^{*} \in X^{*}$ such that there exist sequences $\lambda_{k} \downarrow 0, x_{k} \stackrel{\psi}{\rightarrow} \bar{x}$, and $x_{k}^{*} \in \widehat{\partial} \psi\left(x_{k}\right)$ with $\lambda_{k} x_{k}^{*} \stackrel{w^{*}}{\rightarrow} x^{*}$, where $x_{k} \stackrel{\psi}{\rightarrow} \bar{x}$ means that $x_{k} \rightarrow \bar{x}$ and $\psi\left(x_{k}\right) \rightarrow \psi(\bar{x})$, and $\lambda_{k} \downarrow 0$ means that $\lambda_{k} \rightarrow 0$ and $\lambda_{k}>0$ for every $k$. This subdifferential structure is particularly important in recognizing the Lipschitz continuity of functions; see, e.g., [7, Theorem 3.52] and [10, Theorem 9.13].

The paper is organized as follows. Section 2 presents general properties of the directional minimal time function (1). In Section 3, we study generalized differentiation properties of the function that involve various kinds of subdifferential structures in convex and nonconvex 
settings. Although some results in Section 2 and 3 can be derived from [8], we provide detailed simplified proofs for the convenience of the reader. Section 4 is devoted to the study of Lipschitzian properties using both direct and generalized differentiation approaches. Finally, in Section 5, we apply the results from the previous sections to study location problems (3).

Throughout the paper, we use the following standing assumptions unless otherwise stated: $\Omega$ is a nonempty closed subset of a real normed linear space $X ; v$ is a nonzero vector in $X$. Moreover, when there is no risk of confusion, we will use $T$ instead of $T_{v}(\cdot, \Omega)$ and $\varphi$ instead of $\varphi_{v}(\cdot, \Omega)$. For a nonzero vector $v \in X$, we will use the following notations:

$$
\begin{aligned}
& \text { cone }\{v\}:=\{\lambda v \mid \lambda \geq 0\}=\mathbb{R}_{+} v, \\
& \text { span }\{v\}:=\{\lambda v \mid \lambda \in \mathbb{R}\}=\mathbb{R} v, \\
& \{v\}^{+}:=\left\{x^{*} \in X^{*} \mid\left\langle x^{*}, v\right\rangle \geq 0\right\}, \\
& \{v\}^{\perp}:=\left\{x^{*} \in X^{*} \mid\left\langle x^{*}, v\right\rangle=0\right\} .
\end{aligned}
$$

\section{General Properties}

Let us start with simple representations of the domain and the epigraph of the directional minimal time function (1). Recall that the recession cone of $\Omega$ is given by

$$
\Omega_{\infty}:=\{u \in X \mid \omega+\lambda u \in \Omega \text { for all } \omega \in \Omega \text { and for all } \lambda \geq 0\} .
$$

Proposition 2.1 The domain of the directional minimal time function (1) is given by

$$
\operatorname{dom} T=\Omega-\text { cone }\{v\} .
$$

Suppose further that $v \in \Omega_{\infty}$. Then

$$
\begin{gathered}
\operatorname{dom} T=\Omega-\operatorname{span}\{v\}, \\
\text { epi } T=\{(x, t) \in X \times \mathbb{R} \mid t \geq 0, x+t v \in \Omega\},
\end{gathered}
$$

and

$$
T(x)=\max \{\varphi(x), 0\} \quad \forall x \in X
$$

Proof: By the definition,

$$
\begin{aligned}
\operatorname{dom} T & =\{x \in X \mid T(x)<\infty\} \\
& =\{x \in X \mid x+t v \in \Omega \text { for some } t \geq 0\} \\
& =\{x \in X \mid x \in \Omega-t v \text { for some } t \geq 0\} \\
& =\Omega-\operatorname{cone}\{v\} .
\end{aligned}
$$

Assume that $v \in \Omega_{\infty}$. For any $x \in \Omega-\operatorname{span}\{v\}$, one has $x=\omega-\lambda v$, where $\omega \in \Omega$ and $\lambda \in \mathbb{R}$. If $\lambda \geq 0$, then $x \in \Omega-$ cone $\{v\}=\operatorname{dom} T$. In the case $\lambda<0$, since $v \in \Omega_{\infty}$, one has $x=\omega+(-\lambda) v \in \Omega \subseteq \operatorname{dom} T$. Thus, (4) holds in this case. The proof for (5) is straightforward. 
The inequality $\geq$ in (6) (even for arbitrary $v$ ) is obvious. Let $\max \{\varphi(x), 0\}<\lambda$. By the definition of $\varphi$, there exists $t \in(-\infty, \lambda)$ such that $x+t v \in \Omega$. Then $x+\lambda v=x+t v+(\lambda-t) v \in$ $\Omega+\mathbb{R}_{+} v=\Omega$, and so $T(x) \leq \lambda$. It follows that $T(x) \leq \max \{\varphi(x), 0\}$.

Remark 2.2 The scalarization function associated with $\Omega$ and $v$ was introduced by Gerstewitz (Tammer) and Iwanow [6] as in (2). This function has been used extensively in vector optimization; see [11] and the references therein. Two important properties of $\varphi_{v}(\cdot ; \Omega)$ are:

$$
\varphi_{v}(x+t v ; \Omega)=\varphi_{v}(x ; \Omega)-t \text { for all } x \in X, t \in \mathbb{R},
$$

and

$$
\varphi_{v}(\cdot ; \Omega)=\varphi_{v}\left(\cdot ; \Omega+\mathbb{R}_{+} v\right) .
$$

However, $T_{v}(\cdot ; \Omega) \geq T_{v}\left(\cdot ; \Omega+\mathbb{R}_{+} v\right)$ in general. This can be seen by taking $X=\mathbb{R}^{2}$, $\Omega=[-1,1] \times[-1,1]$, and $v=(1,0)$.

Proposition 2.3 The infimum in the directional minimal time function (1) always attains for any $x \in \operatorname{dom} T$. That means

$$
\Pi_{v}(x ; \Omega):=x+T(x) v \in \Omega
$$

for all $x \in \operatorname{dom} T$. In fact, $\Pi_{v}(x ; \Omega) \in$ bd $\Omega$ for all $x$ with $T(x) \in(0, \infty)$.

Proof: Let $x \in \operatorname{dom} T$ and let $t:=T(x)$. Then there exists a sequence $t_{k} \rightarrow t$ with $t_{k} \in[0, \infty)$ and $x+t_{k} v \in \Omega$ for every $k$. Thus, $x+t v \in \Omega$ since $\Omega$ is closed. Suppose $t \in(0, \infty)$ and $\Pi_{v}(x ; \Omega) \notin$ bd $\Omega$. Then $\Pi_{v}(x ; \Omega) \in$ int $\Omega$, so there exists $\delta>0$ such that $x+t v+\mathbb{B}(0 ; \delta) \subseteq \Omega$ and $\frac{\delta}{\|v\|}<t$. Then $x+t v-\frac{\delta}{\|v\|} v \in \Omega$. This implies $t=T(x) \leq t-\frac{\delta}{\|v\|}$, which is a contradiction.

For any $x \in \operatorname{dom} T$, the element $\Pi_{v}(x ; \Omega)=x+T(x) v$ is called the projection from $x$ to $\Omega$ with respect to the directional minimal time function (1).

Proposition 2.4 For any $\alpha \in \mathbb{R}$, define

$$
\mathcal{L}_{\alpha}:=\{x \in X \mid T(x) \leq \alpha\} \text { and } \mathcal{L}_{\alpha}^{<}:=\{x \in X \mid T(x)<\alpha\} .
$$

Then

$$
\mathcal{L}_{\alpha}=\Omega-[0, \alpha] v \text { and } \mathcal{L}_{\alpha}^{<}=\Omega-[0, \alpha) v \text { for } \alpha \geq 0 .
$$

The equality $T(x)=0$ holds if and only if $x \in \Omega$. Moreover, $T$ is lower semicontinuous.

Proof: The proof of (8) is obvious. Suppose $x \in \Omega$. Then $x+0 v \in \Omega$, so $T(x) \leq 0$, and hence $T(x)=0$. Now suppose $T(x)=0$. Then $x+T(x) v=x \in \Omega$. Since $\mathcal{L}_{\alpha}$ is closed for every $\alpha \in \mathbb{R}\left(\mathcal{L}_{\alpha}\right.$ being empty for $\left.\alpha<0\right)$, the function $T$ is lower semicontinuous.

Remark 2.5 Because $T$ is lsc, in the case $v \in \Omega_{\infty}$, by (6), we have that $T$ and $\varphi$ coincide on a neighborhood of $x$ for any $x \in X$ with $T(x)>0$. This shows that the properties of $T$ proved at elements $x \in X$ with $T(x)>0$ can be extended to similar properties of $\varphi$ [having in view also (7)]. 
Proposition 2.6 The function $T$ is convex if and only if $\Omega$ is convex.

Proof: Suppose that $\Omega$ is convex. Fix $x_{1}, x_{2} \in \operatorname{dom} T$ and $\lambda \in(0,1)$. Then

$$
x_{1}+T\left(x_{1}\right) v \in \Omega \text { and } x_{2}+T\left(x_{2}\right) v \in \Omega .
$$

Since $\Omega$ is convex, this implies

$$
\lambda x_{1}+(1-\lambda) x_{2}+\left[\lambda T\left(x_{1}\right)+(1-\lambda) T\left(x_{2}\right)\right] v \in \Omega .
$$

Thus,

$$
T\left(\lambda x_{1}+(1-\lambda) x_{2}\right) \leq \lambda T\left(x_{1}\right)+(1-\lambda) T\left(x_{2}\right) .
$$

Conversely, if $T$ is convex, then $\{x \in X \mid T(x) \leq 0\}=\Omega$ is convex.

We are now going to study the strict convexity of $T$. For $a, b \in X$, let

$$
[a, b]:=\{t a+(1-t) b \mid t \in[0,1]\}
$$

be the line segment connecting the two points.

A set $\Omega$ is called strictly convex if for any $x_{1}, x_{2} \in \Omega, x_{1} \neq x_{2}$, and for any $t \in(0,1)$, one has $t x_{1}+(1-t) x_{2} \in$ int $\Omega$.

Proposition 2.7 Suppose that $[a, b] \subseteq \operatorname{dom} T \backslash \Omega$ and $b-a \notin \operatorname{span}\{v\}$. If $\Omega$ is strictly convex, then $T$ is strictly convex on $[a, b]$.

Proof: Suppose by contradiction that $x_{1}, x_{2} \in[a, b], x_{1} \neq x_{2}, \alpha \in(0,1)$ and

$$
T\left(\alpha x_{1}+(1-\alpha) x_{2}\right)=\alpha T\left(x_{1}\right)+(1-\alpha) T\left(x_{2}\right)>0 .
$$

Then

$$
\begin{aligned}
\Pi_{v}\left(\alpha x_{1}+(1-\alpha) x_{2} ; \Omega\right) & =\alpha x_{1}+(1-\alpha) x_{2}+T\left(\alpha x_{1}+(1-\alpha) x_{2}\right) v \\
& =\alpha\left(x_{1}+T\left(x_{1}\right) v\right)+(1-\alpha)\left(x_{2}+T\left(x_{2}\right) v\right) \\
& =\alpha \Pi_{v}\left(x_{1} ; \Omega\right)+(1-\alpha) \Pi_{v}\left(x_{2} ; \Omega\right) .
\end{aligned}
$$

Since $x_{1}, x_{2} \in \operatorname{dom} T$ and $x_{1}-x_{2} \notin \mathbb{R} v$, one has that $\Pi_{v}\left(x_{1} ; \Omega\right) \neq \Pi_{v}\left(x_{2} ; \Omega\right)$. Consequently,

$$
\alpha \Pi_{v}\left(x_{1} ; \Omega\right)+(1-\alpha) \Pi_{v}\left(x_{2} ; \Omega\right) \in \operatorname{int} \Omega,
$$

which is a contradiction since the projection must belong to the boundary of $\Omega$ by Proposition 2.3.

Proposition 2.8 Fix $\bar{x} \in \operatorname{dom} T$. One always has

$$
T(\bar{x}+\lambda v)=T(\bar{x})-\lambda \text { for any } 0 \leq \lambda \leq T(\bar{x}) .
$$

For $\lambda>0$, suppose further that $\bar{x}-\gamma v \notin \Omega$ for every $\gamma \in(0, \lambda]$. Then

$$
T(\bar{x}-\lambda v)=T(\bar{x})+\lambda .
$$

Moreover,

$$
T(x+t v) \geq \max \{T(x)-t, 0\} \text { for all } x \in X \text { and } t \geq 0,
$$

and the equality holds if $v \in \Omega_{\infty}$. 
Proof: Since $\bar{x}+T(\bar{x}) v \in \Omega$, one has $\bar{x}+\lambda v+(T(\bar{x})-\lambda) v \in \Omega$. Thus,

$$
T(\bar{x}+\lambda v) \leq T(\bar{x})-\lambda<\infty .
$$

Let us show that

$$
T(\bar{x}) \leq \lambda+T(\bar{x}+\lambda v) .
$$

Indeed, one can assume $t:=T(\bar{x}+\lambda v)<\infty$. Then

$$
\bar{x}+\lambda v+t v \in \Omega .
$$

This implies $T(\bar{x}) \leq \lambda+t$, and the result follows. From (11), one has $T(\bar{x})-\lambda \leq T(\bar{x}+\lambda v)$, and hence (9) holds.

Let us now prove the second equality under the assumption that $\bar{x}-\gamma v \notin \Omega$ for every $\gamma \in(0, \lambda]$. Let $\bar{y}:=\bar{x}-\lambda v$. Then $\bar{x}=\bar{y}+\lambda v$. From (11), one has

$$
T(\bar{y})=T(\bar{x}-\lambda v) \leq T(\bar{x})+\lambda<\infty .
$$

This implies $\bar{y} \in \operatorname{dom} T$. In this case, we can easily see that $0<\lambda \leq T(\bar{y})$. Indeed, let $\eta:=T(\bar{y})$. Then $\bar{y}+\eta v=\bar{x}-\lambda v+\eta v=\bar{x}-(\lambda-\eta) v \in \Omega$. This implies $\lambda-\eta \notin(0, \lambda]$, so $\lambda \leq \eta=T(\bar{y})$. Applying (9), one has $T(\bar{x})=T(\bar{y})-\lambda=T(\bar{x}-\lambda v)-\lambda$, and the second equality follows.

The inequality (10) follows from (11). Let us prove that the equality holds if $v \in \Omega_{\infty}$. There is nothing to prove if $T(x)=\infty$. Consider two cases $T(x)>t$ and $T(x) \leq t$. In the first case, one has

$$
x+t v+(T(x)-t) v=x+T(x) v \in \Omega .
$$

Thus, $T(x+t v) \leq T(x)-t$, and the conclusion holds. In the second case, one has $x+t v=$ $x+T(x) v+(t-T(x)) v \in \Omega+\Omega_{\infty}=\Omega$. Thus, $T(x+t v)=0$, and the conclusion also holds.

Corollary 2.9 Assume that there exist $\bar{x} \in \Omega$ and $\bar{t}>0$ such that $\bar{x}+\bar{t} v \in \Omega$ but $[\bar{x}, \bar{x}+\bar{t} v] \nsubseteq$ $\Omega$. Then there exists $t \in[0, \bar{t})$ such that $T$ is finite and not continuous at $\bar{x}+t v$.

Proof: By hypothesis, there exists $t_{0} \in(0, \bar{t})$ such that $\bar{x}+t_{0} v \notin \Omega$. Considering the largest interval $I \subseteq[0, \bar{t}]$ containing $t_{0} \in I$ such that $\bar{x}+s v \notin \Omega$ for every $s \in I$, we may assume that $I=(0, \bar{t})$. Thus, for every $\lambda \in(0, \bar{t})$, we have that $\bar{x}+\bar{t} v-\gamma v \notin \Omega$ for every $\gamma \in(0, \lambda]$. Using the previous proposition, we get $T(\bar{x}+\bar{t} v-\lambda v)=T(\bar{x}+\bar{t} v)+\lambda=\lambda$, and so

$$
\lim _{t \rightarrow 0_{+}} T(\bar{x}+t v)=\lim _{\lambda \rightarrow \bar{t}_{-}} T(\bar{x}+\bar{t} v-\lambda v)=\bar{t} \neq T(\bar{x})=0 .
$$

Therefore, $T$ is not continuous at $\bar{x}$.

The next result provides a sufficient condition and a necessary condition for the continuity of $T$ at some $\bar{x} \in \operatorname{dom} T$.

Proposition 2.10 Let $\bar{x} \in \operatorname{dom} T$. If $\bar{x}+(T(\bar{x}), T(\bar{x})+\gamma) v \subseteq$ int $\Omega$ for some $\gamma>0$, then $T$ is continuous at $\bar{x}$. Suppose additionally that $v \in \Omega_{\infty}$. Then $T$ is continuous at $\bar{x}$ if and only if $\bar{x}+(T(\bar{x}), \infty) v \subseteq$ int $\Omega$. 
Proof: Fix any number $\lambda$ such that $T(\bar{x})<\lambda$. We can assume that $\lambda<T(\bar{x})+\gamma$. Then $\bar{x}+\lambda v \in$ int $\Omega$. Choose $\delta>0$ such that

$$
\bar{x}+\lambda v+\delta \mathbb{B} \subseteq \Omega .
$$

For any $x \in \bar{x}+\delta \mathbb{B}$, one has $T(x) \leq \lambda$. Thus, $T$ is upper semicontinuous at $\bar{x}$, so it is continuous at this point.

Suppose that $v \in \Omega_{\infty}$ and $T$ is continuous at $\bar{x}$. Consider $\lambda>T(\bar{x})$. Since $T$ is continuous at $\bar{x}$, there exists $\delta>0$ such that whenever $x \in V:=\bar{x}+\delta \mathbb{B}$, one has $T(x)<\lambda$. This implies $x+\lambda v \in \Omega$, so $x \in \Omega-\lambda v$. Thus, $V \subseteq \Omega-\lambda v$ or $\lambda v+V=\bar{x}+\lambda v+\delta \mathbb{B} \subseteq \Omega$. Therefore, $\bar{x}+\lambda v \in$ int $\Omega$.

Example 2.11 Let $\Omega:=\left\{(x, y) \in \mathbb{R}^{2} \mid y=0\right\}$ and let $v=(0,1)$. Then $T$ is continuous at $\bar{x}=(0,-1)$, but $\bar{x}+(T(\bar{x}), \infty) v \nsubseteq$ int $\Omega$.

We end this section with other properties of the directional minimal time function which will be used in the next sections.

Proposition 2.12 For two nonempty closed subsets $A$ and $B$ of $X$, the following hold:

(1) If $A \subseteq B$, then $T_{v}(x, B) \leq T_{v}(x, A)$ for all $x \in X$.

(2) $T_{v}(x+y ; A+B) \leq T_{v}(x ; A)+T_{v}(y ; B)$ for all $x, y \in X$.

Proof: (1) It is obvious if $T_{v}(x ; A)=\infty$. Suppose $t:=T_{v}(x ; A)<\infty$. Then

$$
x+t v \in A \subseteq B .
$$

Thus, $T_{v}(x ; B) \leq t=T_{v}(x ; A)$.

(2) The conclusion if obvious if $T_{v}(x ; A)=\infty$ or $T_{v}(y ; B)=\infty$. In the other case, let $s:=T_{v}(x ; A)$ and $t:=T_{v}(x ; B)$. Then

$$
x+t v \in A \text { and } y+t v \in B .
$$

Thus, $x+y+(s+t) v \in A+B$. This implies

$$
T_{v}(x+y ; A+B) \leq s+t=T_{v}(x ; A)+T_{v}(y ; B) .
$$

The proof is now complete.

\section{Generalized Differentiation Properties}

In this section, we are going to study generalized differentiation properties of the directional minimal time function (1). Various subdifferential structures of variational analysis will be employed to study the function. The results from this section will be important for the study of Lipschitz continuity of the function in Section 4 and for applications to location problems in Section 5. 


\subsection{Fenchel conjugate and subgradients in the sense of convex analysis}

For a function $\psi: X \rightarrow(-\infty, \infty]$, recall that the Fenchel conjugate of $\psi$ is an extended real-valued function on $X^{*}$ defined by

$$
\psi^{*}\left(x^{*}\right):=\sup \left\{\left\langle x^{*}, x\right\rangle-\psi(x) \mid x \in X\right\} .
$$

Let us start with a formula for representing the Fenchel conjugate of the directional minimal time function (1) in terms of the support function of $\Omega$ defined on $X^{*}$ by

$$
\sigma_{\Omega}\left(x^{*}\right):=\sup \left\{\left\langle x^{*}, \omega\right\rangle \mid \omega \in \Omega\right\} .
$$

Proposition 3.1 The function $T$ is a lsc proper function and

$$
T^{*}\left(x^{*}\right)= \begin{cases}\sigma_{\Omega}\left(x^{*}\right), & \text { if }\left\langle x^{*},-v\right\rangle \leq 1, \\ \infty, & \text { otherwise. }\end{cases}
$$

Proof: The fact that $T$ is lower semicontinuous has been proved in Proposition 2.4. It is proper since $\Omega \subseteq$ dom $T$. We have the following

$$
\begin{aligned}
T^{*}\left(x^{*}\right) & =\sup \left\{\left\langle x^{*}, x\right\rangle-T(x) \mid x \in \operatorname{dom} T\right\} \\
& =\sup \left\{\left\langle x^{*}, x\right\rangle-t \mid t \geq 0, x+t v \in \Omega\right\} \\
& =\sup \left\{\left\langle x^{*}, \omega\right\rangle+t\left[\left\langle x^{*},-v\right\rangle-1\right] \mid t \geq 0, \omega \in \Omega\right\} \\
& =\sup \left\{\left\langle x^{*}, \omega\right\rangle \mid \omega \in \Omega\right\}+\sup \left\{t\left(\left\langle x^{*},-v\right\rangle-1\right) \mid t \geq 0\right\} .
\end{aligned}
$$

The formula then follows easily.

Theorem 3.2 Let $\bar{x} \in \operatorname{dom} T$, where $\Omega$ is convex. Then

$$
\partial T(\bar{x})=\left\{\begin{array}{l}
N(\bar{x}, \Omega) \cap\left\{x^{*} \in X^{*} \mid\left\langle v, x^{*}\right\rangle \geq-1\right\}, \text { if } \bar{x} \in \Omega, \\
N(\bar{x}+T(\bar{x}) v, \Omega) \cap\left\{x^{*} \in X^{*} \mid\left\langle v, x^{*}\right\rangle=-1\right\}, \text { otherwise. }
\end{array}\right.
$$

Moreover, if $\bar{x} \in \Omega$ and $v \in \Omega_{\infty}$, then

$$
\partial T(\bar{x})=\left\{x^{*} \in X^{*} \mid-1 \leq\left\langle x^{*}, v\right\rangle \leq 0\right\} \cap N(\bar{x} ; \Omega) .
$$

Proof: Consider $x^{*} \in \partial T(\bar{x})$. Then $T(\bar{x})+T^{*}\left(x^{*}\right)=\left\langle\bar{x}, x^{*}\right\rangle$. By Proposition 3.1,

$$
\left\langle v, x^{*}\right\rangle \geq-1 \text { and } T(\bar{x})+\sigma_{\Omega}\left(x^{*}\right)=\left\langle\bar{x}, x^{*}\right\rangle .
$$

Since $\bar{x}+T(\bar{x}) v \in \Omega$, the following holds

$$
\left\langle\bar{x}, x^{*}\right\rangle \geq T(\bar{x})+\left\langle\bar{x}+T(\bar{x}) v, x^{*}\right\rangle .
$$

It follows that

$$
T(\bar{x})\left[1+\left\langle v, x^{*}\right\rangle\right] \leq 0 .
$$

Because $T(\bar{x}) \geq 0$ and $1+\left\langle v, x^{*}\right\rangle \geq 0$, one has

$$
T(\bar{x})\left[1+\left\langle v, x^{*}\right\rangle\right]=0 .
$$


If $\bar{x} \in \Omega$, then $\sigma_{\Omega}\left(x^{*}\right)=\left\langle\bar{x}, x^{*}\right\rangle$, and so $x^{*} \in N(\bar{x}, \Omega)$. If $\bar{x} \notin \Omega$, then $T(\bar{x})>0$, and so $1+\left\langle v, x^{*}\right\rangle=0$. It follows that

$$
\sigma_{\Omega}\left(x^{*}\right)=\left\langle\bar{x}+T(\bar{x}) v, x^{*}\right\rangle
$$

and $x^{*} \in N(\bar{x}+T(\bar{x}) v, \Omega)$. Thus, the inclusion $\subseteq$ holds in (12). Conversely, if $\bar{x} \in \Omega$ and $x^{*} \in N(\bar{x}, \Omega) \cap\left\{x^{*} \in X^{*} \mid\left\langle v, x^{*}\right\rangle \geq-1\right\}$, then

$$
T(\bar{x})\left[1+\left\langle v, x^{*}\right\rangle\right]=0 \text { and } \sigma_{\Omega}\left(x^{*}\right)=\left\langle\bar{x}, x^{*}\right\rangle .
$$

Thus, $T(\bar{x})+T^{*}\left(x^{*}\right)=\left\langle\bar{x}, x^{*}\right\rangle$, and so $x^{*} \in \partial T(\bar{x})$. If $\bar{x} \notin \Omega$ and $x^{*} \in N(\bar{x}+T(\bar{x}) v, \Omega) \cap$ $\left\{x^{*} \in X^{*} \mid\left\langle v, x^{*}\right\rangle=-1\right\}$, then

$$
T(\bar{x})\left[1+\left\langle v, x^{*}\right\rangle\right]=0 \text { and } \sigma_{\Omega}\left(x^{*}\right)=\left\langle\bar{x}+T(\bar{x}) v, x^{*}\right\rangle .
$$

It follows that $T(\bar{x})+T^{*}\left(x^{*}\right)=\left\langle\bar{x}, x^{*}\right\rangle$, and again $x^{*} \in \partial T(\bar{x})$.

Under the condition $v \in \Omega_{\infty}$, one sees easily that $\left\langle x^{*}, v\right\rangle \leq 0$ for every $x^{*} \in N(\bar{x} ; \Omega)$. Thus, equality (13) follows.

Proposition 3.3 Let $\bar{x} \in \Omega$, where $\Omega$ is convex and $X$ is a Banach space. Then

$$
\partial^{\infty} T(\bar{x})=N(\bar{x}, \Omega) \cap\{v\}^{+}=N(\bar{x}, \operatorname{dom} T) .
$$

Moreover, if $v \in \Omega_{\infty}$, then

$$
\partial^{\infty} T(\bar{x})=N(\bar{x}, \Omega) \cap\{v\}^{\perp}=N(\bar{x}, \operatorname{dom} T) .
$$

Proof: Fix any $x^{*} \in \partial^{\infty} T(\bar{x})$. Then there exist sequences $x_{k} \stackrel{T}{\rightarrow} \bar{x}, \lambda_{k} \downarrow 0, \lambda_{k} x_{k}^{*} \stackrel{w^{*}}{\rightarrow} x^{*}$ such that $x_{k}^{*} \in \partial T\left(x_{k}\right)$. Let $\tilde{x}_{k}:=x_{k}+T\left(x_{k}\right) v \in \Omega$. By Theorem $3.2, x_{k}^{*} \in N\left(\tilde{x}_{k} ; \Omega\right)$ and $\left\langle x_{k}^{*},-v\right\rangle \leq 1$ (the equality holds if $x_{k} \notin \Omega$ ). Then $\lambda_{k} x_{k}^{*} \in N\left(\tilde{x}_{k} ; \Omega\right)$, and $\left(\lambda_{k} x_{k}^{*}\right)$ is a bounded sequence as $X$ is a Banach space. Since $\tilde{x}_{k}:=x_{k}+T\left(x_{k}\right) v \rightarrow \bar{x}$, one has $x^{*} \in N(\bar{x} ; \Omega)$. Moreover, $\left\langle\lambda_{k} x_{k}^{*},-v\right\rangle \leq \lambda_{k}$, and hence $\left\langle x^{*},-v\right\rangle \leq 0$. It follows that $x^{*} \in\{v\}^{+}$. Now fix any $x^{*} \in N(\bar{x}, \Omega) \cap\{v\}^{+}$. Then $k x^{*} \in N(\bar{x} ; \Omega)$ and $\left\langle k x^{*},-v\right\rangle \leq 0<1$ for every $k$. By Theorem $3.2, k x^{*} \in \partial T(\bar{x})$, and hence $x^{*} \in \frac{1}{k} \partial T(\bar{x})$. By definition, $x^{*} \in \partial^{\infty} T(\bar{x})$.

We have seen that $\operatorname{dom} T=\Omega-\mathbb{R}_{+} v$, and so

$$
\begin{aligned}
x^{*} \in N(\bar{x}, \operatorname{dom} T) & \Leftrightarrow\left\langle x-t v-\bar{x}, x^{*}\right\rangle \leq 0 \forall x \in \Omega, t \geq 0 \\
& \Leftrightarrow\left\langle x-\bar{x}, x^{*}\right\rangle \leq 0 \forall x \in \Omega \text { and }\left\langle v, x^{*}\right\rangle \geq 0 .
\end{aligned}
$$

This implies $N(\bar{x}, \operatorname{dom} T)=N(\bar{x}, \Omega) \cap\{v\}^{+}$.

The proof for the second equalities under the condition $v \in \Omega_{\infty}$ follows from the last observation in the proof of Theorem 3.2.

Proposition 3.4 Let $\bar{x} \in \operatorname{dom} T \backslash \Omega$, where $\Omega$ is convex and $X$ is a Banach space. Then

$$
\partial^{\infty} T(\bar{x}) \subseteq N(\bar{x}+T(\bar{x}) v, \Omega) \cap\{v\}^{\perp} .
$$

Moreover, if $\partial T(\bar{x})$ is nonempty, then equality holds. 
Proof: Let $x^{*} \in \partial^{\infty} T(\bar{x})$. Then there exist sequences $x_{k} \stackrel{T}{\rightarrow} \bar{x}, \lambda_{k} \downarrow 0, \lambda_{k} x_{k}^{*} \stackrel{w^{*}}{\rightarrow} x^{*}$ such that $x_{k}^{*} \in \partial T\left(x_{k}\right)$ for $k \geq 1$. Since $T\left(x_{k}\right) \rightarrow T(\bar{x})>0$, we may assume that $T\left(x_{k}\right)>0$ for $k \geq 1$. Let $\tilde{x}_{k}:=x_{k}+T\left(x_{k}\right) v \in \Omega$. Clearly, $\tilde{x}_{k} \rightarrow \tilde{x}:=\bar{x}+T(\bar{x}) v \in \Omega$. By Theorem 3.2,

$$
x_{k}^{*} \in N\left(\tilde{x}_{k} ; \Omega\right) \text { and }\left\langle v, x_{k}^{*}\right\rangle=-1 .
$$

Because $X$ is a Banach space, we have that $\left(\lambda_{k} x_{k}^{*}\right)$ is bounded. From the inequality $\left\langle x-\tilde{x}_{k}, x_{k}^{*}\right\rangle \leq 0$ for $k \geq 1$ and $x \in \Omega$, one has

$$
\left\langle x-\tilde{x}_{k}, \lambda_{k} x_{k}^{*}\right\rangle \leq 0
$$

This implies $\left\langle x-\tilde{x}, x^{*}\right\rangle \leq 0$ for $x \in \Omega$. As $\left\langle v, \lambda_{k} x_{k}^{*}\right\rangle=-\lambda_{k} \rightarrow 0$, we also have that $\left\langle v, x^{*}\right\rangle=0$. Therefore, $x^{*} \in N(\tilde{x}, \Omega) \cap\{v\}^{\perp}$.

Assume now that $\partial T(\bar{x}) \neq \emptyset$ and fix $x_{0}^{*} \in \partial T(\bar{x})$. By Theorem 3.2, we have that $x_{0}^{*} \in N(\bar{x}+T(\bar{x}) v, \Omega)$ and $\left\langle v, x_{0}^{*}\right\rangle=-1$. Take $x^{*} \in N(\bar{x}+T(\bar{x}) v, \Omega) \cap\{v\}^{\perp}$. Then

$$
x_{k}^{*}:=x_{0}^{*}+k x^{*} \in N(\bar{x}+T(\bar{x}) v, \Omega) \text { and }\left\langle v, x_{k}^{*}\right\rangle=-1 .
$$

Thus, $x_{k}^{*} \in \partial T(\bar{x})$. Taking $x_{k}:=\bar{x}$ and $\lambda_{k}:=1 / k$, we obtain that $x^{*} \in \partial^{\infty} T(\bar{x})$.

Example 3.5 Let $\Omega=\mathbb{B}(0 ; \sqrt{8})$ in $\mathbb{R}^{2}$ with the Euclidean norm and let $v=(1,1)$. For $\bar{x}=(-2,-2) \in \Omega$, using the formula from Theorem 3.2 , one has

$$
\partial T(\bar{x})=\{(t, t) \mid-1 / 2 \leq t \leq 0\}
$$

For $\bar{x}=(2,2)$, one has

$$
\partial T(\bar{x})=\{(t, t) \mid t \geq 0\}
$$

For $\bar{x}=(-3,-3) \notin \Omega$, one has

$$
\partial T(\bar{x})=\{(-1 / 2,-1 / 2)\}
$$

We end this section with a result referring to the scalarization function $\varphi$ given in (2). The result is immediate from Proposition 3.4 and Theorem 3.2, using Remark 2.5.

Corollary 3.6 Let $\bar{x} \in X$ satisfy $\varphi(\bar{x}) \in \mathbb{R}$, where $\Omega$ is convex. Assume that $v \in \Omega_{\infty}$. Then

$$
\partial \varphi(\bar{x})=N(\bar{x}+\varphi(\bar{x}) v ; \Omega) \cap\left\{x^{*} \in X^{*} \mid\left\langle x^{*}, v\right\rangle=-1\right\} .
$$

Moreover, if $X$ is a Banach space, then

$$
\partial^{\infty} \varphi(\bar{x}) \subseteq N(\bar{x}+\varphi(\bar{x}) v ; \Omega) \cap\{v\}^{\perp},
$$

and the equality holds if $\partial \varphi(\bar{x}) \neq \emptyset$. 


\subsection{Dini-Hadamard directional derivatives and subgradients}

Let $\psi: X \rightarrow(-\infty, \infty]$ be a function and let $\bar{x} \in$ dom $\psi$. The Dini-Hadamard directional derivative of the function at $\bar{x}$ in the direction $u$ is given by

$$
\psi^{\prime}(\bar{x}, u):=\liminf _{t \rightarrow 0^{+}, v \rightarrow u} \frac{\psi(\bar{x}+t v)-\psi(\bar{x})}{t} .
$$

If $\psi$ is Lipschitz continuous around $\bar{x}$, the Dini-Hadamard directional derivative coincides with the (lower) Dini directional derivative

$$
\psi^{\prime}(\bar{x} ; h):=\liminf _{t \rightarrow 0^{+}} \frac{\psi(\bar{x}+t h)-\psi(\bar{x})}{t} .
$$

Let $\bar{x} \in \Omega$. The Bouligand contingent cone to $\Omega$ at $\bar{x}$, denoted by $K(\bar{x} ; \Omega)$, is the set of $d \in X$ such that there exist sequences $t_{k} \rightarrow 0^{+}$and $d_{k} \rightarrow d$ with $\bar{x}+t_{k} d_{k} \in \Omega$ for every $k$. It is well-known that $K(\bar{x} ; \Omega)$ is a closed cone. Moreover, $K((x, \psi(x))$; epi $\psi)=\operatorname{epi~} \psi^{\prime}(x ; \cdot)$ if $x \in \operatorname{dom} \psi$.

We also define the Dini-Hadamard normal cone

$$
N^{-}(\bar{x} ; \Omega):=\left\{x^{*} \in X^{*} \mid\left\langle x^{*}, d\right\rangle \leq 0 \text { for all } d \in K(\bar{x} ; \Omega)\right\},
$$

and the Dini-Hadamard subdifferential of $\psi$ at $\bar{x}$

$$
\partial^{-} \psi(\bar{x})=\left\{x^{*} \in X^{*} \mid\left\langle x^{*}, h\right\rangle \leq \psi^{\prime}(\bar{x} ; h) \text { for all } h \in X\right\}
$$

Lemma 3.7 Let $\bar{x} \in \Omega$. Then

$$
T^{\prime}(\bar{x} ; h)=T_{v}(h ; K(\bar{x} ; \Omega)) .
$$

Proof: Let $\bar{x} \in \Omega$. Set $\lambda:=T^{\prime}(\bar{x}, u)$; because $T(x) \geq T(\bar{x})=0$ for every $x \in X$, we have that $\lambda \geq 0$. If $\lambda=+\infty$, we have clearly that $T_{v}(u, K(\bar{x}, \Omega)) \leq T^{\prime}(\bar{x}, u)$. Assume that $\lambda<\infty$. Then there exist sequences $u_{k} \rightarrow u$ and $t_{k} \rightarrow 0^{+}$such that

$$
\lambda_{k}:=t_{k}^{-1}\left[T\left(\bar{x}+t_{k} u_{k}\right)-T(\bar{x})\right]=t_{k}^{-1} T\left(\bar{x}+t_{k} u_{k}\right) \rightarrow \lambda .
$$

In particular, we may assume that $\lambda_{k} \in \mathbb{R}_{+}$for $k \geq 1$. It follows that $\bar{x}+t_{k}\left(u_{k}+\lambda_{k} v\right) \in \Omega$, whence $u+\lambda v \in K(\bar{x}, \Omega)$, and so $T_{v}(u, K(\bar{x}, \Omega)) \leq \lambda$. Therefore, $T_{v}(u, K(\bar{x}, \Omega)) \leq T^{\prime}(\bar{x}, u)$.

Conversely, set $\lambda:=T_{v}(u, K(\bar{x}, \Omega)) \in[0, \infty]$. Assume that $\lambda<\infty$. Then $u+\lambda v \in$ $K(\bar{x}, \Omega)$, and so there exist sequences $u_{k}^{\prime} \rightarrow u+\lambda v$ and $t_{k} \rightarrow 0^{+}$such that $\bar{x}+t_{k} u_{k}^{\prime}=$ $\bar{x}+t_{k}\left(u_{k}^{\prime}-\lambda v\right)+t_{k} \lambda v \in \Omega$ for $k \geq 1$. Then $T\left(\bar{x}+t_{k}\left(u_{k}^{\prime}-\lambda v\right)\right) \leq t_{k} \lambda$. Since $T(\bar{x})=0$ and $\left(u_{k}^{\prime}-\lambda v\right) \rightarrow u$, we obtain that $T^{\prime}(\bar{x}, u) \leq \lambda$. Thus, $T^{\prime}(\bar{x}, u) \leq T_{v}(u, K(\bar{x}, \Omega))$ for every $u \in X$.

Theorem 3.8 For any $\bar{x} \in \Omega$, one has

$$
\partial^{-} T(\bar{x})=\left\{x^{*} \mid\left\langle x^{*},-v\right\rangle \leq 1\right\} \cap N^{-}(\bar{x} ; \Omega) .
$$


Proof: Fix any $x^{*} \in \partial^{-} T(\bar{x})$. By the definition and Lemma 3.7,

$$
\left\langle x^{*}, h\right\rangle \leq T^{\prime}(\bar{x} ; h)=T_{v}(h ; K(\bar{x} ; \Omega)) \text { for all } h \in X .
$$

For $h=-v$, one has $\left\langle x^{*},-v\right\rangle \leq T_{v}(-v ; K(\bar{x} ; \Omega)) \leq 1$ since $0 \in K(\bar{x} ; \Omega)$. Moreover, for all $h \in K(\bar{x} ; \Omega)$, one has

$$
\left\langle x^{*}, h\right\rangle \leq T_{v}(h ; K(\bar{x} ; \Omega))=0 .
$$

Thus, $x^{*} \in N^{-}(\bar{x} ; \Omega)$.

Now suppose that $\left\langle x^{*},-v\right\rangle \leq 1$ and $x^{*} \in N^{-}(\bar{x} ; \Omega)$. For any $h \in X$, let us show that

$$
\left\langle x^{*}, h\right\rangle \leq T^{\prime}(\bar{x} ; h) .
$$

The inequality holds obviously if $T^{\prime}(\bar{x} ; h)=\infty$. Consider the case where

$$
t:=T_{v}(h ; K(\bar{x} ; \Omega))<\infty .
$$

Then $h+t v \in K(\bar{x} ; \Omega)$. Thus, $\left\langle x^{*}, h+t v\right\rangle \leq 0$. This implies

$$
\left\langle x^{*}, h\right\rangle \leq t\left\langle x^{*},-v\right\rangle \leq t=T_{v}(h ; K(\bar{x} ; \Omega))=T^{\prime}(\bar{x} ; h) .
$$

Therefore, $x^{*} \in \partial^{-} T(\bar{x})$, and (14) has been proved.

Lemma 3.9 Let $\bar{x} \in \operatorname{dom} T \backslash \Omega$ and let $\tilde{x}:=\Pi_{v}(\bar{x} ; \Omega)$. Then

$$
T^{\prime}(\bar{x}, u) \leq \varphi_{v}(u, K(\tilde{x}, \Omega)) \text { for every } u \in X .
$$

Moreover, equality holds for those $u \in X$ with $T^{\prime}(\bar{x}, u) \neq-\infty$. This equality also holds if $v \in \Omega_{\infty}$.

Proof: Assume that $\varphi_{v}(u, K(\tilde{x}, \Omega))<\lambda \in \mathbb{R}$. Then there exists $\mu \in(-\infty, \lambda)$ such that $u+\mu v \in K(\tilde{x}, \Omega)$, and so there exist $\left(u_{k}^{\prime}\right) \rightarrow u+\mu v$ and $\left(t_{k}\right) \rightarrow 0^{+}$such that $\tilde{x}+t_{k} u_{k}^{\prime}=$ $\bar{x}+t_{k}\left(u_{k}^{\prime}-\mu v\right)+\left(T(\bar{x})+t_{k} \mu\right) v \in \Omega$ for $k \geq 1$. Since $T(\bar{x})>0$, there exists $k_{0} \geq 1$ such that $T(\bar{x})+t_{k} \mu>0$ for $k \geq k_{0}$. It follows that

$$
T\left(\bar{x}+t_{k}\left(u_{k}^{\prime}-\mu v\right)\right) \leq T(\bar{x})+t_{k} \mu .
$$

Since $u_{k}^{\prime}-\mu v \rightarrow u$, one has $T^{\prime}(\bar{x}, u) \leq \mu<\lambda$. Thus, $T^{\prime}(\bar{x}, u) \leq \varphi_{v}(u, K(\tilde{x}, \Omega))$.

Assume now that $\lambda:=T^{\prime}(\bar{x}, u) \in \mathbb{R}$. Then there exist sequences $u_{k} \rightarrow u$ and $t_{k} \rightarrow 0^{+}$ such that $\lambda_{k}:=t_{k}^{-1}\left[T\left(\bar{x}+t_{k} u_{k}\right)-T(\bar{x})\right] \rightarrow \lambda$. In particular, we may assume that $\lambda_{k} \in \mathbb{R}$ for $k \geq 1$. It follows that

$$
\bar{x}+T(\bar{x}) v+t_{k}\left(u_{k}+\lambda_{k} v\right)=\bar{x}+t_{k} u_{k}+\left[T(\bar{x})+t_{k} \lambda_{k}\right] v \in \Omega .
$$

Since $\left(u_{k}+\lambda_{k} v\right) \rightarrow u+\lambda v$, we have that $u+\lambda v \in K(\bar{x}+T(\bar{x}) v, \Omega)$, and so $\varphi_{v}(u, K(\tilde{x}, \Omega)) \leq \lambda$. The conclusion follows.

The proof of the equality in the case where $v \in \Omega_{\infty}$ is left for the readers.

A function $\psi: X \rightarrow(-\infty, \infty]$ is called calm at $\bar{x} \in \operatorname{dom} \psi$ if there exist constants $\ell \geq 0$ and $\delta>0$ such that

$$
|\psi(x)-\psi(\bar{x})| \leq \ell\|x-\bar{x}\| \text { for all } x \in \mathbb{B}(\bar{x} ; \delta) .
$$


We also say that $\psi$ is lower calm at $\bar{x}$ if there exist constants $\ell \in \mathbb{R}$ and $\delta>0$ such that

$$
\psi(x)-\psi(\bar{x}) \geq \ell\|x-\bar{x}\| \text { for all } x \in \mathbb{B}(\bar{x} ; \delta) .
$$

It is obvious that if $\psi$ is Lipschitz continuous around $\bar{x}$, then it is (lower) calm at every point in a neighborhood of $\bar{x}$.

Corollary 3.10 Let $\bar{x} \in \operatorname{dom} T \backslash \Omega$ and let $\tilde{x}:=\Pi_{v}(\bar{x} ; \Omega)$. Suppose that $T$ is lower calm at $\bar{x}$. Then

$$
T^{\prime}(\bar{x}, u)=\varphi_{v}(u ; K(\tilde{x}, \Omega)) \text { for every } u \in X \text {. }
$$

Proof: By definition,

$$
T^{\prime}(\bar{x} ; u)=\liminf _{t \rightarrow 0^{+}, v \rightarrow u} \frac{T(\bar{x}+t v)-T(\bar{x})}{t} \geq \liminf _{t \rightarrow 0^{+}, v \rightarrow u} \frac{\ell t\|v\|}{t}=\ell\|u\|>-\infty,
$$

where $\ell$ is a constant. The equality then follows directly from Lemma 3.9.

Theorem 3.11 Let $\bar{x} \in \operatorname{dom} T \backslash \Omega$ and let $\tilde{x}:=\Pi_{v}(\bar{x} ; \Omega)$. Then

$$
\partial^{-} T(\bar{x}) \subseteq\left\{x^{*} \in X^{*} \mid\left\langle x^{*},-v\right\rangle=1\right\} \cap N^{-}(\tilde{x} ; \Omega) .
$$

The opposite inclusion holds if $T$ is lower calm at $\bar{x}$.

Proof: Fix any $x^{*} \in \partial^{-} T(\bar{x})$. By Lemma 3.9,

$$
\left\langle x^{*}, h\right\rangle \leq T^{\prime}(\bar{x} ; h) \leq \varphi_{v}(h, K(\tilde{x}, \Omega)) \text { for all } h \in X .
$$

A similar argument to the proof of Theorem 3.8 gives us $\left\langle x^{*},-v\right\rangle \leq 1$ and $x^{*} \in N^{-}(\tilde{x} ; \Omega)$.

We also have, using Proposition 2.8, that

$$
\left\langle x^{*}, v\right\rangle \leq T^{\prime}(\bar{x}, v) \leq \liminf _{t \rightarrow 0^{+}} \frac{T(\bar{x}+t v)-T(\bar{x})}{t}=\liminf _{t \rightarrow 0^{+}} \frac{T(\bar{x})-t-T(\bar{x})}{t}=-1 .
$$

Thus, $\left\langle x^{*},-v\right\rangle=1$.

Let us prove the opposite inclusion under the lower calmness; hence $T^{\prime}(\bar{x}, h)>-\infty$ for all $h \in X$. Fix any $x^{*} \in X^{*}$ such that $\left\langle x^{*},-v\right\rangle=1$ and $x^{*} \in N^{-}(\tilde{x} ; \Omega)$. We will show that

$$
\left\langle x^{*}, h\right\rangle \leq T^{\prime}(\bar{x} ; h) \text { for all } h \in X \text {. }
$$

Fix any $h \in X$. The inequality obviously holds when $T^{\prime}(\bar{x} ; h)=+\infty$; so, assume that $T^{\prime}(\bar{x} ; h) \in \mathbb{R}$. By Corollary 3.10,

$$
h+T^{\prime}(\bar{x} ; h) v \in K(\tilde{x}, \Omega) .
$$

It follows that

$$
\left\langle x^{*}, h+T^{\prime}(\bar{x} ; h) v\right\rangle \leq 0 .
$$

Thus,

$$
\left\langle x^{*}, h\right\rangle \leq T^{\prime}(\bar{x} ; h)\left\langle x^{*},-v\right\rangle .
$$

Therefore, $\left\langle x^{*}, h\right\rangle \leq T^{\prime}(\bar{x} ; h)$, and hence $x^{*} \in \partial^{-} T(\bar{x})$.

The result referring to $\varphi_{v}(\cdot ; \Omega)$ which corresponds to Corollary 3.10 and Theorem 3.11 is the following. 
Corollary 3.12 Assume that $v \in \Omega_{\infty}$ and $\bar{x} \in X$ is such that $\varphi(\bar{x}) \in \mathbb{R}$, where $\varphi:=$ $\varphi_{v}(\cdot ; \Omega)$. If $\varphi$ is lower calm at $\bar{x}$, then

$$
\varphi^{\prime}(\bar{x} ; u)=\varphi_{v}(u ; K(\bar{x}+\varphi(\bar{x}) v, \Omega)) \text { for every } u \in X
$$

and

$$
\partial^{-} \varphi(\bar{x})=N^{-}(\bar{x}+\varphi(\bar{x}) v ; \Omega) \cap\left\{x^{*} \in X^{*} \mid\left\langle x^{*},-v\right\rangle=1\right\} .
$$

\subsection{Hölder and Fréchet subgradients}

Let $\psi: X \rightarrow(-\infty, \infty]$ be an extended real-valued function and let $\bar{x} \in \operatorname{dom} \psi$. Given $s>0$, an element $x^{*} \in X^{*}$ is called an $s$-Hölder subgradient of the function $\psi$ at $\bar{x}$ if there exist $\delta>0$ and $\sigma>0$ such that

$$
\left\langle x^{*}, x-\bar{x}\right\rangle \leq \psi(x)-\psi(\bar{x})+\sigma\|x-\bar{x}\|^{1+s} \text { for all } x \in \mathbb{B}(\bar{x} ; \delta) .
$$

The set of all $s$-Hölder subgradients of the function at $\bar{x}$ is called the $s$-Hölder subdifferential of the function at this point and is denoted by $\partial_{s} \psi(\bar{x})$.

Similarly, the $s$-Hölder normal cone to a set $\Omega$ at $\bar{x} \in \Omega$ is the set of all $x^{*} \in X^{*}$ such that there exist $\delta>0$ and $\sigma>0$ such that

$$
\left\langle x^{*}, x-\bar{x}\right\rangle \leq \sigma\|x-\bar{x}\|^{1+s} \text { for all } x \in \mathbb{B}(\bar{x} ; \delta) \cap \Omega .
$$

In the case where $s=1$ and $X$ is a Hilbert space, these structures reduce to the proximal subdifferential and proximal normal cone, respectively; see [4].

Proposition 3.13 For any $\bar{x} \in \Omega$, one has the following representation of $s$-Hölder subgradients:

$$
\partial_{s} T(\bar{x})=\left\{x^{*} \in X^{*} \mid\left\langle x^{*},-v\right\rangle \leq 1\right\} \cap N_{s}(\bar{x} ; \Omega) .
$$

Suppose additionally that $v \in \Omega_{\infty}$. Then

$$
\partial_{s} T(\bar{x})=\left\{x^{*} \in X^{*} \mid-1 \leq\left\langle x^{*}, v\right\rangle \leq 0\right\} \cap N_{s}(\bar{x} ; \Omega) .
$$

Proof: Fix any $x^{*} \in \partial_{s} T(\bar{x})$. Then there exist $\delta>0$ and $\sigma>0$ such that

$$
\left\langle x^{*}, x-\bar{x}\right\rangle \leq T(x)-T(\bar{x})+\sigma\|x-\bar{x}\|^{1+s} \text { for all } x \in \mathbb{B}(\bar{x} ; \delta) .
$$

Since $T(x)=0$ for all $x \in \Omega$, one has

$$
\left\langle x^{*}, x-\bar{x}\right\rangle \leq \sigma\|x-\bar{x}\|^{1+s} \text { for all } x \in \mathbb{B}(\bar{x} ; \delta) \cap \Omega .
$$

This implies $x^{*} \in N_{s}(\bar{x} ; \Omega)$. Since $\bar{x}-t v \in \mathbb{B}(\bar{x} ; \delta)$ for $t>0$ sufficiently small, one has

$$
\left\langle x^{*},-t v\right\rangle \leq T(\bar{x}-t v)+\sigma t^{1+s}\|v\|^{1+s} \leq t+\sigma t^{1+s}\|v\|^{1+s} .
$$

It follows that

$$
\left\langle x^{*},-v\right\rangle \leq 1+\sigma t^{s}\|v\|^{1+s} .
$$

Letting $t \rightarrow 0$, one has $\left\langle x^{*},-v\right\rangle \leq 1$. The inclusion $\subseteq$ in (15) has been proved. 
Now fix any $x^{*} \in X^{*}$ such that $\left\langle x^{*},-v\right\rangle \leq 1$ and $x^{*} \in N_{s}(\bar{x} ; \Omega)$. Then there exist $\delta>0$ and $\sigma>0$ such that

$$
\left\langle x^{*}, x-\bar{x}\right\rangle \leq \sigma\|x-\bar{x}\|^{1+s} \text { for all } x \in \mathbb{B}(\bar{x} ; \delta) \cap \Omega \text {. }
$$

Suppose by contradiction that $x^{*} \notin \partial_{s} T(\bar{x})$. Then there exist sequences $\sigma_{k} \rightarrow \infty$ and $x_{k} \rightarrow \bar{x}$ such that

$$
\left\langle x^{*}, x_{k}-\bar{x}\right\rangle>T\left(x_{k}\right)+\sigma_{k}\left\|x_{k}-\bar{x}\right\|^{1+s} \geq T\left(x_{k}\right)=: t_{k} .
$$

This implies $t_{k} \rightarrow 0$ as $k \rightarrow \infty$ and $x_{k} \neq \bar{x}$ for every $k$. Moreover, $t_{k} \leq\left\|x^{*}\right\|\left\|x_{k}-\bar{x}\right\|$. Let $\tilde{x}_{k}:=x_{k}+t_{k} v \in \Omega$. For sufficiently large $k$, one has

$$
\left\langle x^{*}, \tilde{x}_{k}-\bar{x}\right\rangle \leq \sigma\left\|\tilde{x}_{k}-\bar{x}\right\|^{1+s} \leq \sigma\left(\left\|x_{k}-\bar{x}\right\|+t_{k}\|v\|\right)^{1+s} \leq \sigma\left(1+\left\|x^{*}\right\|\|v\|\right)^{1+s}\left\|x_{k}-\bar{x}\right\|^{1+s} .
$$

We also have

$$
\left\langle x^{*}, \tilde{x}_{k}-\bar{x}\right\rangle=\left\langle x^{*}, x_{k}-\bar{x}\right\rangle+t_{k}\left\langle x^{*}, v\right\rangle \geq\left\langle x^{*}, x_{k}-\bar{x}\right\rangle-t_{k}>\sigma_{k}\left\|x_{k}-\bar{x}\right\|^{1+s} .
$$

It follows that for sufficiently large $k$,

$$
\sigma_{k}\left\|x_{k}-\bar{x}\right\|^{1+s}<\sigma\left(1+\left\|x^{*}\right\|\|v\|\right)^{1+s}\left\|x_{k}-\bar{x}\right\|^{1+s},
$$

which implies $\sigma_{k}<\sigma\left(1+\left\|x^{*}\right\|\|v\|\right)^{1+s}$. We have arrived at a contradiction since $\sigma_{k} \rightarrow \infty$.

Suppose now that $v \in \Omega_{\infty}$. Using $x:=\bar{x}+t v \in \mathbb{B}(\bar{x} ; \delta)$ for $t>0$ sufficiently small, we arrive at $\left\langle x^{*}, v\right\rangle \leq 0$, and hence (16) holds. The proof is now complete.

Theorem 3.14 For any $\bar{x} \in \operatorname{dom} T \backslash \Omega$, one has the following representation of s-Hölder subgradients:

$$
\partial_{s} T(\bar{x}) \subseteq\left\{x^{*} \in X^{*} \mid\left\langle x^{*}, v\right\rangle=-1\right\} \cap N_{s}(\tilde{x} ; \Omega),
$$

where $\tilde{x}:=\Pi_{v}(\bar{x} ; \Omega)$. Suppose further that $T$ is lower calm at $\bar{x}$. Then

$$
\partial_{s} T(\bar{x})=\left\{x^{*} \in X^{*} \mid\left\langle x^{*}, v\right\rangle=-1\right\} \cap N_{s}(\tilde{x} ; \Omega) .
$$

Proof: Fix any $x^{*} \in \partial_{s} T(\bar{x})$. Then there exist $\delta>0$ and $\sigma>0$ such that

$$
\left\langle x^{*}, x-\bar{x}\right\rangle \leq T(x)-T(\bar{x})+\sigma\|x-\bar{x}\|^{1+s}
$$

for all $x \in \mathbb{B}(\bar{x} ; \delta)$. Since $\Omega$ is closed, we can assume without loss of generality that $\mathbb{B}(\bar{x} ; \delta) \cap \Omega=\emptyset$. Choose $t>0$ sufficiently small so that $\bar{x}-t v \in \mathbb{B}(\bar{x} ; \delta)$. Then (using also Proposition 2.8)

$$
\left\langle x^{*},(\bar{x}-t v)-\bar{x}\right\rangle \leq T(\bar{x}-t v)-T(\bar{x})+\sigma t^{1+s}\|v\|^{1+s} \leq T(\bar{x})+t-T(\bar{x})+\sigma t^{1+s}\|v\|^{1+s} .
$$

This implies

$$
\left\langle x^{*},-v\right\rangle \leq 1+\sigma t^{s}\|v\|^{1+s} .
$$

Thus, $\left\langle x^{*},-v\right\rangle \leq 1$. Using $\bar{x}+t v$ in a similar way, one has $\left\langle x^{*},-v\right\rangle \geq 1$. 
Fix any $x \in \Omega$ with $\|x-\tilde{x}\|<\delta$. Then $\|x-T(\bar{x}) v-\bar{x}\|<\delta$. Let $t:=T(\bar{x})$. Using $x-t v+t v \in \Omega$, one has

$$
\begin{aligned}
\left\langle x^{*}, x-\tilde{x}\right\rangle & =\left\langle x^{*}, x-t v-\bar{x}\right\rangle \\
& \leq T(x-t v)-T(\bar{x})+\sigma\|x-\tilde{x}\|^{1+s} \\
& \leq t-T(\bar{x})+\sigma\|x-\tilde{x}\|^{1+s} \\
& =\sigma\|x-\tilde{x}\|^{1+s} .
\end{aligned}
$$

Thus, $x^{*} \in N_{s}(\tilde{x} ; \Omega)$.

Let us prove the opposite inclusion under the calmness of $T$. Let $\ell, \delta>0$ be such that

$$
T(x)-T(\bar{x}) \geq-\ell\|x-\bar{x}\| \quad \forall x \in B(\bar{x}, \delta) .
$$

Take $x^{*} \in N_{s}(\tilde{x} ; \Omega)$ with $\left\langle x^{*}, v\right\rangle=-1$. Then there exist $\sigma>0$ and $\delta^{\prime}>0$ such that

$$
\left\langle x^{*}, u-\tilde{x}\right\rangle \leq \sigma\|u-\tilde{x}\|^{1+s} \quad \forall u \in B\left(\tilde{x}, \delta^{\prime}\right) .
$$

Assume that $x^{*} \notin \partial_{s} T(\bar{x})$. Then there exists a sequence $\left(x_{k}\right) \subseteq B(\bar{x}, \delta)$ such that $x_{k} \rightarrow \bar{x}$ and

$$
\left\langle x^{*}, x_{k}-\bar{x}\right\rangle>T\left(x_{k}\right)-T(\bar{x})+k\left\|x_{k}-\bar{x}\right\|^{1+s} \quad \forall k \geq 1 .
$$

From (17) and (19) we obtain that $\left|T\left(x_{k}\right)-T(\bar{x})\right| \leq \ell^{\prime}\left\|x_{k}-\bar{x}\right\|$, where $\ell^{\prime}:=\max \left(\ell,\left\|x^{*}\right\|\right)$, and so $T\left(x_{k}\right) \rightarrow T(\bar{x})$. Set $u_{k}:=x_{k}+T\left(x_{k}\right) v \in \Omega$; clearly, $u_{k} \rightarrow \tilde{x}$, and so $u_{k} \in B\left(\tilde{x}, \delta^{\prime}\right)$ for $k \geq k_{0}$ with $k_{0} \geq 1$ fixed. It follows that for $k \geq k_{0}$ we have

$$
\begin{aligned}
\left\langle x^{*}, x_{k}-\bar{x}\right\rangle-\left[T\left(x_{k}\right)-T(\bar{x})\right] & =\left\langle x^{*}, u_{k}-\tilde{x}\right\rangle \leq \sigma\left\|u_{k}-\tilde{x}\right\|^{1+s} \\
& =\sigma\left\|x_{k}-\bar{x}+\left[T\left(x_{k}\right)-T(\bar{x})\right] v\right\|^{1+s} \\
& \leq \sigma\left(1+\ell^{\prime}\|v\|\right)^{1+s}\left\|x_{k}-\bar{x}\right\|^{1+s} .
\end{aligned}
$$

Using (19) we get

$$
k\left\|x_{k}-\bar{x}\right\|^{1+s}<\left\langle x^{*}, x_{k}-\bar{x}\right\rangle-\left[T\left(x_{k}\right)-T(\bar{x})\right] \leq \sigma\left(1+\ell^{\prime}\|v\|\right)^{1+s}\left\|x_{k}-\bar{x}\right\|^{1+s}
$$

for $k \geq k_{0}$, whence the contradiction $k<\sigma\left(1+\ell^{\prime}\|v\|\right)^{1+s}$ for every $k \geq k_{0}$. Hence $x^{*} \in$ $\partial_{s} T(\bar{x})$.

Similar proofs yield the representations below for Fréchet subgradients of the directional minimal time function (1) in both in-set case and out-of-set case.

Proposition 3.15 For any $\bar{x} \in \Omega$, one has the following representation of Fréchet subgradients:

$$
\widehat{\partial} T(\bar{x})=\left\{x^{*} \in X^{*} \mid\left\langle x^{*},-v\right\rangle \leq 1\right\} \cap \widehat{N}(\bar{x} ; \Omega) .
$$

Suppose additionally that $v \in \Omega_{\infty}$. Then

$$
\widehat{\partial} T(\bar{x})=\left\{x^{*} \in X^{*} \mid-1 \leq\left\langle x^{*}, v\right\rangle \leq 0\right\} \cap \widehat{N}(\bar{x} ; \Omega) .
$$


Theorem 3.16 For any $\bar{x} \in \operatorname{dom} T \backslash \Omega$, one has the following representation of Fréchet subgradients:

$$
\widehat{\partial} T(\bar{x}) \subseteq\left\{x^{*} \in X^{*} \mid\left\langle x^{*}, v\right\rangle=-1\right\} \cap \widehat{N}(\tilde{x} ; \Omega),
$$

where $\tilde{x}:=\Pi_{v}(\bar{x} ; \Omega)$. Suppose further that $T_{v}(\cdot ; \Omega)$ is lower calm at $\bar{x}$. Then

$$
\widehat{\partial} T(\bar{x})=\left\{x^{*} \in X^{*} \mid\left\langle x^{*}, v\right\rangle=-1\right\} \cap \widehat{N}(\tilde{x} ; \Omega) .
$$

The result referring to $\varphi_{v}(\cdot ; \Omega)$ which corresponds to Theorems 3.14 and 3.16 is the following.

Corollary 3.17 Assume that $v \in \Omega_{\infty}, \bar{x} \in X$ is such that $\varphi(\bar{x}) \in \mathbb{R}$, where $\varphi:=\varphi_{v}(\cdot ; \Omega)$, and $s>0$. Then

$$
\partial_{s} \varphi(\bar{x}) \subseteq\left\{x^{*} \in X^{*} \mid\left\langle x^{*}, v\right\rangle=-1\right\} \cap N_{s}(\bar{x}+\varphi(\bar{x}) v ; \Omega),
$$

and

$$
\widehat{\partial} \varphi(\bar{x}) \subseteq\left\{x^{*} \in X^{*} \mid\left\langle x^{*}, v\right\rangle=-1\right\} \cap \widehat{N}(\bar{x}+\varphi(\bar{x}) v ; \Omega) .
$$

Moreover, if $\varphi$ is lower calm at $\bar{x}$, then equalities hold in the previous two inclusions.

\subsection{Limiting Subgradients}

Theorem 3.18 For any $\bar{x} \in \Omega$, one has the following representation of limiting subgradients:

$$
\partial T(\bar{x})=\left\{x^{*} \in X^{*} \mid\left\langle x^{*},-v\right\rangle \leq 1\right\} \cap N(\bar{x} ; \Omega) .
$$

Proof: Fix any $x^{*} \in \partial T(\bar{x})$. Then there exist sequences $x_{k}^{*} \stackrel{w^{*}}{\longrightarrow} x^{*}$ and $x_{k} \stackrel{T}{\rightarrow} \bar{x}$ with $x_{k}^{*} \in \widehat{\partial} T\left(x_{k}\right)$. Let $\tilde{x}_{k}:=\Pi_{v}\left(x_{k} ; \Omega\right)$. Then $\tilde{x}_{k} \stackrel{\Omega}{\rightarrow} \bar{x}$ and $x_{k}^{*} \in \widehat{N}\left(\tilde{x}_{k} ; \Omega\right)$. This implies $x^{*} \in N(\bar{x} ; \Omega)$. In both cases: $x_{k} \in \Omega$ and $x_{k} \notin \Omega$, we always have $\left\langle x_{k}^{*},-v\right\rangle \leq 1$. Thus $\left\langle x^{*},-v\right\rangle \leq 1$.

Let us prove the opposite inclusion. Fix $x^{*} \in X^{*}$ with $\left\langle x^{*},-v\right\rangle \leq 1$ and $x^{*} \in N(\bar{x} ; \Omega)$. Then there exist sequences $x_{k}^{*} \stackrel{w^{*}}{\rightarrow} x^{*}$ and $x_{k} \stackrel{\Omega}{\rightarrow} \bar{x}$ with $x_{k}^{*} \in \widehat{N}\left(x_{k} ; \Omega\right)$. Define $\gamma_{k}:=$ $\left\langle x_{k}^{*},-v\right\rangle$. If $\gamma_{k} \leq 1$ for a subsequence (without relabeling), then $x_{k}^{*} \in \widehat{\partial} T\left(x_{k}\right)$, and hence $x^{*} \in \partial T(\bar{x})$. So we can assume $\gamma_{k}>1$ for every $k$. Clearly, $\gamma_{k} \rightarrow \gamma:=\left\langle x^{*},-v\right\rangle=1$. Let $\tilde{x}_{k}^{*}:=\frac{x_{k}^{*}}{\gamma_{k}}$. Then $\tilde{x}_{k}^{*} \in \widehat{\partial} T\left(x_{k}\right)$. So again, $x^{*} \in \partial T(\bar{x})$.

We say that $\Omega$ satisfies property $P$ around $\bar{x}$ with a radius $r>0$ if there exists a neighborhood $V$ of $\bar{x}$ such that $x-t v \notin \Omega$ for all $t \in(0, r]$ and for all $x \in V \cap$ bd $\Omega$.

For example, if $\Omega$ is the epigraph of a continuous function $\psi: X \rightarrow(-\infty, \infty]$ and $v=(0,1)$, where 0 is the zero element of $X$, then property $P$ is satisfied.

Theorem 3.19 For any $\bar{x} \in \operatorname{dom} T \backslash \Omega$, one has the following upper estimate of limiting subgradients:

$$
\partial T(\bar{x}) \subseteq\left\{x^{*} \in X^{*} \mid\left\langle x^{*}, v\right\rangle=-1\right\} \cap N(\tilde{x} ; \Omega),
$$

where $\tilde{x}:=\Pi_{v}(\bar{x} ; \Omega)$. Suppose further that $T$ is lower calm around $\bar{x}$. Then

$$
\partial T(\bar{x})=\left\{x^{*} \in X^{*} \mid\left\langle x^{*}, v\right\rangle=-1\right\} \cap N(\tilde{x} ; \Omega),
$$

under the assumption that $\Omega$ satisfies condition $P$ around $\tilde{x}$ with the radius $r=T(\bar{x})$. 
Proof: Fix any $x^{*} \in \partial T(\bar{x})$. Then there exist sequences $x_{k} \rightarrow \bar{x}$ with $T\left(x_{k}\right) \rightarrow T(\bar{x})$ and $x_{k}^{*} \stackrel{w^{*}}{\rightarrow} x^{*}$ with $x_{k}^{*} \in \widehat{\partial} T\left(x_{k}\right)$. Under the assumption made, $\left\langle x_{k}^{*}, v\right\rangle=-1$ and $x_{k}^{*} \in \widehat{N}\left(\tilde{x}_{k} ; \Omega\right)$ for sufficiently large $k$, where $\tilde{x}_{k}:=x_{k}+T\left(x_{k}\right) v \in \Omega$ (see Theorem 3.16). Then $\left\langle x^{*}, v\right\rangle=-1$. Clearly, $\tilde{x}_{k} \stackrel{\Omega}{\rightarrow} \tilde{x}$. Thus, $x^{*} \in \widehat{N}(\tilde{x} ; \Omega)$. The first inclusion has been proved.

Let us prove the opposite inclusion. Fix any $x^{*} \in X^{*}$ with $\left\langle x^{*}, v\right\rangle=-1$ and $x^{*} \in$ $\widehat{N}(\tilde{x} ; \Omega)$. Then there exist $\tilde{x}_{k} \stackrel{\Omega}{\rightarrow} \tilde{x}$ and $x_{k}^{*} \in \widehat{N}\left(\tilde{x}_{k} ; \Omega\right)$ with $x_{k}^{*} \stackrel{w^{*}}{\rightarrow} x^{*}$. Let $\gamma_{k}:=-\left\langle x_{k}^{*}, v\right\rangle$. Then $\gamma_{k} \rightarrow 1$ as $k \rightarrow \infty$. For sufficiently large $k$, let $\tilde{x}_{k}^{*}:=\frac{x_{k}^{*}}{\gamma_{k}}$. Then $\left\langle\tilde{x}_{k}^{*}, v\right\rangle=-1$ and $\tilde{x}_{k}^{*} \in \widehat{N}\left(\tilde{x}_{k} ; \Omega\right)$ by the cone property of the Fréchet normal cone. Clearly, $\tilde{x}_{k}$ belongs to the boundary of $\Omega$. Let $x_{k}:=\tilde{x}_{k}-r v$. Under the $\mathrm{P}$ property, and using Proposition 2.8, we have that $T\left(x_{k}\right)=r$ and $\Pi\left(x_{k} ; \Omega\right)=\tilde{x}_{k}$. Thus, by Theorem 3.16, $\tilde{x}_{k}^{*} \in \widehat{\partial} T\left(x_{k}\right)$. Since $x_{k} \stackrel{T}{\rightarrow} \bar{x}$, one has $x^{*} \in \partial T(\bar{x})$.

Theorem 3.20 For $\bar{x} \in \Omega$, one has

$$
\partial^{\infty} T(\bar{x})=\{v\}^{+} \cap N(\bar{x} ; \Omega)
$$

Proof: The proof of $\subseteq$ follows directly from the definition of singular subgradients and the proof of Theorem 3.19. Let us prove the opposite inclusion. Fix any $x^{*} \in\{v\}^{+} \cap N(\bar{x} ; \Omega)$. Then there exist $x_{k} \stackrel{\Omega}{\rightarrow} \bar{x}, x_{k}^{*} \stackrel{w^{*}}{\rightarrow} x^{*}$ such that $x_{k}^{*} \in \widehat{N}\left(x_{k} ; \Omega\right)$; clearly $x_{k} \stackrel{T}{\rightarrow} \bar{x}$. Set $\mu_{k}:=\left\langle x_{k}^{*}, v\right\rangle \rightarrow\left\langle x^{*}, v\right\rangle \geq 0$. If $\mu_{k} \geq 0$ for a subsequence (without relabeling), then $\left\langle k x_{k}^{*}, v\right\rangle \geq 0 \geq-1$, and hence $k x_{k}^{*} \in \widehat{\partial} T\left(x_{k}\right)$. This implies $x^{*} \in \partial^{\infty} T(\bar{x})$. In the contrary case $\mu_{k}<0$ for every $k$, and so $\lambda_{k}:=-\mu_{k} \downarrow 0$. Then $\left\langle\frac{x_{k}^{*}}{\lambda_{k}}, v\right\rangle=-1$, and so $\frac{x_{k}^{*}}{\lambda_{k}} \in \widehat{\partial} T\left(x_{k}\right)$. Then, by definition, again $x^{*} \in \partial^{\infty} T(\bar{x})$.

The proof of the theorem below is also straightforward.

Theorem 3.21 For any $\bar{x} \in \operatorname{dom} T \backslash \Omega$, one has

$$
\partial^{\infty} T(\bar{x}) \subseteq\{v\}^{\perp} \cap N(\bar{x}+T(\bar{x}) v ; \Omega) .
$$

\section{Lipschitz properties of directional minimal time functions}

In this section, we are going to study Lipschitz properties of the directional minimal time function (1). Necessary and sufficient conditions for globally Lipschitz property and locally Lipschitz property/Lipschitz continuity will be established.

Proposition 4.1 Suppose that $v \in$ int $\Omega_{\infty}$. Then $T$ is globally Lipschitz with Lipschitz constant

$$
\ell:=\inf \left\{r^{-1} \mid r>0, \mathbb{B}(v ; r) \subseteq \Omega_{\infty}\right\}=\frac{1}{\operatorname{dist}\left(v, \operatorname{bd} \Omega_{\infty}\right)} .
$$

Proof: Let $K:=\Omega_{\infty}$. We first show that $\operatorname{dom} T_{v}(\cdot ; \Omega)=X$. Indeed, since $v \in \operatorname{int} K$,

$$
\operatorname{dom} T_{v}(\cdot ; \Omega)=\Omega-\operatorname{span}\{v\}=\Omega+[K-\operatorname{span}\{v\}]=\Omega+X=X
$$


It follows from Proposition 2.12 (2) that

$$
T_{v}(x ; \Omega+K) \leq T_{v}(y ; \Omega)+T_{v}(x-y ; K) .
$$

Then

$$
T_{v}(x ; \Omega) \leq T_{v}(y ; \Omega)+T_{v}(x-y ; K) .
$$

This implies

$$
T_{v}(x ; \Omega)-T_{v}(y ; \Omega) \leq T_{v}(x-y ; K) .
$$

Fix any $r>0$ such that $\mathbb{B}(v ; r) \subseteq K$. Then $\mathbb{B}(0 ; r) \subseteq K-v$. We have the following for $u \notin K$ by the cone property of $K$

$$
\begin{aligned}
T_{v}(u ; K) & =\inf \{t \geq 0 \mid u+t v \in K\} \\
& =\inf \{t>0 \mid u \in t(K-v)\} \\
& \leq \inf \{t>0 \mid u \in t \mathbb{B}(0 ; r)\}=\left\|r^{-1} u\right\| .
\end{aligned}
$$

Since $T_{v}(u ; K)=0 \leq r^{-1}\|u\|$ for $u \in K$, one has

$$
T_{v}(u ; K) \leq r^{-1}\|u\| \quad \forall u \in X .
$$

It follows from (22) that

$$
T_{v}(x ; \Omega)-T_{v}(y ; \Omega) \leq T_{v}(x-y ; K) \leq \ell\|x-y\| .
$$

That implies

$$
\left|T_{v}(y ; \Omega)-T_{v}(x ; \Omega)\right| \leq \ell\|x-y\| .
$$

The proof is now complete.

Proposition 4.2 The function $T$ is finite-valued and Lipschitz if and only if $v \in \operatorname{int} \Omega_{\infty}$.

Proof: Suppose $T$ is finite-valued and Lipschitz. Then $v \in \Omega_{\infty}$. Otherwise, there exist $\bar{x} \in \Omega$ and $t>0$ such that $\bar{x}+t v \notin \Omega$. Because $\bar{x}+t v \in X=\operatorname{dom} T=\Omega-\mathbb{R}_{+} v$, there exists $s>0$ such that $\bar{x}+t v+s v \in \Omega$. Taking $\bar{t}:=t+s$ and using Corollary 2.9, we get some $t_{0} \in[0, \bar{t})$ such that $T$ is not continuous at $\bar{x}+t_{0} v$, contradicting our hypothesis. Therefore, $v \in \Omega_{\infty}$.

Let $\ell \geq 0$ be the Lipschitz constant of $T$, that is

$$
|T(x)-T(y)| \leq \ell\|u-v\| \text { for all } x, y \in X .
$$

We are going to show that $v+\frac{1}{\ell} \mathbb{B} \in \Omega_{\infty}$. Indeed, fix an $e \in \frac{1}{\ell} \mathbb{B}$. Using Proposition 2.8, for any $x \in \Omega$ and $t \geq 0$, one has

$T(x+t(v+e))=T(x+t e+t v)=\max \{T(x+t e)-t, 0\} \leq \max \{T(x)+t \ell\|e\|-t, 0\} \leq 0$.

This implies $x+t(v+e) \in \Omega$. Thus, $v+e \in \Omega_{\infty}$.

The converse follows from Proposition 4.1.

In what follows, we are going to characterize the Lipschitz continuity of the minimal time function (1) using both direct and generalized differentiation approaches. 
Lemma 4.3 Suppose that $T$ is Lipschitz continuous around $\tilde{x}:=\Pi_{v}(\bar{x} ; \Omega)$, where $\bar{x} \in$ $\operatorname{dom} T$. Then $T$ is Lipschitz continuous around $\bar{x}$. Moreover, the converse holds if $v \in \Omega_{\infty}$.

Proof: By hypothesis, there exist $\ell \geq 0, \delta>0$ such that $T$ is finite on $\mathbb{B}(\tilde{x} ; \delta)$ and

$$
|T(x)-T(y)| \leq \ell\|x-y\| \text { for all } x, y \in \mathbb{B}(\tilde{x} ; \delta) .
$$

If $\bar{x} \in \Omega$ the conclusion is obvious. In the contrary case take $0<\varepsilon<\min \{T(\bar{x}), \delta /(1+\|v\|)\}$. Then there exists $\delta^{\prime} \in(0, \varepsilon]$ such that $T(x)>t:=T(\bar{x})-\varepsilon>0$ for every $x \in B\left(\bar{x} ; \delta^{\prime}\right)$. Then for $x \in B\left(\bar{x} ; \delta^{\prime}\right)$ we have that

$$
\|(x+t v)-\tilde{x}\|=\|(x-\bar{x})-\varepsilon v\| \leq \varepsilon(1+\|v\|)<\delta,
$$

and so $x+t v \in \mathbb{B}(\tilde{x} ; \delta)$, and $T(x+t v)=T(x)-t$ (by Proposition 2.8). It follows that

$$
|T(x)-T(y)|=|T(x+t v)+t-T(y+t v)-t|=|T(x+t v)-T(y+t v)| \leq \ell\|x-y\|
$$

for $x, y \in B\left(\bar{x} ; \delta^{\prime}\right)$.

Assume that $v \in \Omega_{\infty}$ and that $T$ is Lipschitz around $\bar{x}$. Let $\bar{x} \notin \Omega$. Using (6) (see also Remark 2.5), we obtain that $\varphi$ is Lipschitz around $\bar{x}$. Using now (7) we obtain that $\varphi$ is Lipschitz around $\tilde{x}$. Using again (6) we get the Lipschitz continuity of $T$. The proof is complete.

Lemma 4.4 Let $\bar{x} \in \operatorname{dom} T$. The function $T$ is Lipschitz continuous around $\bar{x}$ if and only if there exist $\ell \geq 0$ and $\delta>0$ such that

$$
T(x+u) \leq \ell\|u\| \text { for all } u \in \delta \mathbb{B} \text { and } x \in \Omega \cap \mathbb{B}(\bar{x} ; \delta) .
$$

Proof: The implication $\Rightarrow$ is obvious. Assume (23) holds for $\ell \geq 0$ and $\delta>0$. Consider $\delta^{\prime}:=\delta /(2+\ell\|v\|)$ and take $u, u^{\prime} \in \delta^{\prime} \mathbb{B}$. Then $\left\|u-u^{\prime}\right\| \leq 2 \delta^{\prime} \leq \delta$ and $T(\bar{x}+u) \leq \ell \delta^{\prime}$. We may assume that $\left(\ell \delta^{\prime} \geq\right) T(\bar{x}+u) \geq t:=T\left(\bar{x}+u^{\prime}\right)(\geq 0)$. Using Proposition 2.8, we have that $T(\bar{x}+u+t v)=T(\bar{x}+u)-t$. Since $\|u+t v\| \leq \delta^{\prime}+t\|v\| \leq \delta^{\prime}(1+\ell\|v\|) \leq \delta$ and $\bar{x}+u^{\prime}+t v \in \Omega \cap \mathbb{B}(\bar{x} ; \delta)$, using (23) we get

$$
\left|T(\bar{x}+u)-T\left(\bar{x}+u^{\prime}\right)\right|=T(\bar{x}+u)-t=T(\bar{x}+u+t v) \leq \ell\left\|u-u^{\prime}\right\| .
$$

Therefore, $T$ is Lipschitz on $\mathbb{B}\left(\bar{x} ; \delta^{\prime}\right)$ with the same constant $\ell$.

Recall that $\Omega$ is epi-Lipschitz at $\bar{x} \in X$ in the direction $v \neq 0$ if there exists $\delta>0$ such that for all $\omega \in \Omega \cap \mathbb{B}(\bar{x} ; \delta), u \in \mathbb{B}(v ; \delta)$, and $\lambda \in[0, \delta]$, one has $\omega+\lambda u \in \Omega$; see, e.g., $[10,11]$.

Theorem 4.5 Let $\bar{x} \in \Omega$. If $\Omega$ is epi-Lipschitz at $\bar{x}$ in the direction $v$, then $T$ is Lipschitz continuous around $\bar{x}$. Moreover, the converse holds if $v \in \Omega_{\infty}$.

Proof: We only need to show that (23) holds for some $\ell \geq 0$ and $\delta>0$. Under the epiLipschitz condition, we see that $T$ is finite around $\bar{x}$. By contradiction, there exist $v_{k} \rightarrow 0$, $x_{k} \stackrel{\Omega}{\rightarrow} \bar{x}$ and

$$
T\left(x_{k}+v_{k}\right)>k\left\|v_{k}\right\|
$$


Then $v_{k} \neq 0$ for every $k$. With the same notation in the definition of the epi-Lipschitz property, one has that

$$
x_{k}+t(v+e) \in \Omega \text { for } t \in[0, \delta],\|e\| \leq \delta \text { and sufficiently large } k \text {. }
$$

This implies $T\left(x_{k}+t e\right) \leq t$. Thus,

$$
T\left(x_{k}+v_{k}\right)=T\left(x_{k}+\frac{\left\|v_{k}\right\|}{\delta} \frac{\delta v_{k}}{\left\|v_{k}\right\|} ; \Omega\right) \leq \frac{\left\|v_{k}\right\|}{\delta} \text { for sufficiently large } k .
$$

Comparing with (24), we have arrived at a contradiction.

Suppose that $T$ is Lipschitz continuous around $\bar{x} \in \operatorname{dom} T$ and $v \in \Omega_{\infty}$. Suppose by contradiction that $\Omega$ is not epi-Lipschitz around $\bar{x}$ in the direction $v$. Then there exist sequences $x_{k} \stackrel{\Omega}{\rightarrow} \bar{x}, u_{k} \rightarrow 0, t_{k} \rightarrow 0, t_{k}>0$, such that $x_{k}+t_{k}\left(u_{k}+v\right) \notin \Omega$. Then $t_{k}<T\left(x_{k}+t_{k} u_{k}\right)$ because $v \in \Omega_{\infty}$. So

$$
t_{k}<T\left(x_{k}+t_{k} u_{k}\right)=T\left(x_{k}+t_{k} u_{k}\right)-T\left(x_{k}\right) \leq \ell t_{k}\left\|u_{k}\right\|
$$

Since $u_{k} \rightarrow 0$, we have arrived at a contradiction.

Corollary 4.6 Let $\bar{x} \notin \Omega$ such that $T(\bar{x})$ is finite and let $\tilde{x}:=\Pi(\bar{x} ; \Omega)$. If $\Omega$ is epi-Lipschitz at $\tilde{x}$ in the direction $v$, then $T$ is Lipschitz continuous around $\bar{x}$. Moreover, the converse holds if $v \in \Omega_{\infty}$.

Proof: The implication $\Rightarrow$ follows directly from Theorem 4.5 and Lemma 4.3.

Let $v \in \Omega_{\infty}$ and assume that $T$ is Lipschitz continuous around $\bar{x}$. By Lemma 4.3 we have that $T$ is Lipschitz continuous around $\tilde{x} \in \Omega$. By Theorem 4.5 we obtain that $\Omega$ is epi-Lipschitz at $\tilde{x}$ in the direction $v$.

In [11, Theorem 7], a necessary and sufficient condition for the Lipschitz continuity of the scalarization function was proved under the free-disposal condition, that is $\Omega+P=\Omega$ for some closed convex cone $P$ with $v \in P$. Notice that in Theorem 4.5 and Corollary 4.6, the free-disposal condition is not required to prove the sufficient condition for Lipschitz continuity of the directional minimal time function (1). The proof of [11, Theorem 7] is not applicable to our results since it is based on time property from [12, Theorem 2.3.1], which is not satisfied by the directional minimal time function.

In the theorem below, we are able to fully characterize the Lipschitz continuity of the directional minimal time function (1) without using the free-disposal condition. For simplicity, we present our results in finite dimensions.

Theorem 4.7 Let $X$ be a finite dimensional space and let $\bar{x} \in \operatorname{dom} T$.

(1) Assume that $\bar{x} \in \Omega$; then $T$ is Lipschitz continuous around $\bar{x}$ if and only if $\{v\}^{+} \cap$ $N(\bar{x} ; \Omega)=\{0\}$.

(2) Assume that $\bar{x} \notin \Omega$ and set $\tilde{x}:=\Pi(\bar{x} ; \Omega)$. If $\{v\}^{+} \cap N(\tilde{x} ; \Omega)=\{0\}$, then $T$ is Lipschitz continuous around $\bar{x}$. The converse holds true if $v \in \Omega_{\infty}$. 
Proof: (1) On one hand we have $\partial^{\infty} T(\bar{x})=\{v\}^{+} \cap N(\bar{x} ; \Omega)$ by Theorem 3.20. On the other hand, by [10, Theorem 9.13], we have that $T$ is Lipschitz continuous around $\bar{x}$ iff $\partial^{\infty} T(\bar{x})=\{0\}$. The conclusion (i) follows.

(2) In this case we have $\partial^{\infty} T(\bar{x}) \subseteq\{v\}^{+} \cap N(\tilde{x} ; \Omega)$ by Theorem 3.21, and so $T$ is Lipschitz continuous around $\bar{x}$ as in (i). Assume that $v \in \Omega_{\infty}$ and $T$ is Lipschitz continuous around $\bar{x}$. By Lemma 4.3, we have that $T$ is Lipschitz continuous around $\tilde{x} \in \Omega$. By (1) we obtain that $\{v\}^{\perp} \cap N(\tilde{x} ; \Omega)=\{0\}$.

Example 4.8 (1) Let $\Omega:=\left\{(x, y) \in \mathbb{R}^{2}|y \geq-| x \mid\right\}$ and let $v=(0,1)$. Then $T$ is Lipschitz continuous at $\bar{x}=(0,0) \in \Omega$ since the condition $\{v\}^{+} \cap N(\bar{x} ; \Omega)=\{0\}$ is satisfied.

(2) Let $\Omega:=\left\{\left.(x, y) \in \mathbb{R}^{2}|y \geq-| x\right|^{1 / 2}\right\}$ and let $v=(0,1)$. Then $T$ is not Lipschitz continuous at $\bar{x}=(0,0) \in \Omega$ since the condition $\{v\}^{+} \cap N(\bar{x} ; \Omega)=\{0\}$ is violated.

(3) The converse of Theorem 4.7 (2) does not hold true in general. Indeed, let $v=(0,1)$ and

$$
\Omega:=\left\{(x, y) \in \mathbb{R}^{2} \mid y=0\right\} \cup\left\{(x, y) \in \mathbb{R}^{2} \mid x=0, y \geq 0\right\} .
$$

Then $T$ is Lipschitz continuous around $\bar{x}=(0,-2) \notin \Omega$. However,

$$
\{v\}^{\perp} \cap N(\bar{x} ; \Omega)=\left\{(x, y) \in \mathbb{R}^{2} \mid y=0\right\} \neq\{0\} .
$$

\section{Applications to location problems}

In this section, we are going to apply the results obtained previously to study directional location problems. To the best of our knowledge, the location model of this type has not been considered in the literature.

Given the nonempty closed target sets $\Omega_{i}$ for $i=1, \ldots, n$ and $n$ directions $v_{i} \neq 0$ for $i=1, \ldots, n$, and given a nonempty closed constraint set $\Omega_{0}$, find a point $\bar{x} \in \Omega_{0}$ such that the sum of the times to reach the target sets is minimal. The optimization model is

$$
\text { minimize } S(x):=\sum_{i=1}^{n} T_{v_{i}}\left(x ; \Omega_{i}\right) \text { subject to } x \in \Omega_{0} \text {. }
$$

It is clear that

$$
\operatorname{dom} S=\cap_{i=1}^{n} \operatorname{dom} T_{v_{i}}\left(\cdot ; \Omega_{i}\right)=\cap_{i=1}^{n}\left[\Omega_{i}-\operatorname{cone}\left\{v_{i}\right\}\right] .
$$

Proposition 5.1 Suppose dom $S \cap \Omega_{0} \neq \emptyset$. Then the optimization problem (25) has an optimal solution under one of the following conditions:

(1) At least one of the sets among $\Omega_{i}$ for $i=0,1, \ldots, n$ is compact.

(2) $X$ is a reflexive Banach space, $\Omega_{i}$ for $i=0,1, \ldots, n$ are convex and at least one of them is bounded.

Proof: Under the assumptions made, one has

$$
\gamma:=\inf \left\{S(x) \mid x \in \Omega_{0}\right\}<\infty .
$$

Let us first suppose that (1) is satisfied. In the case where the constraint $\Omega_{0}$ is compact, an optimal solution exists by the classical Weierstrass theorem since $S$ is lower semicontinuous. 
Suppose without loss of generality that $\Omega_{1}$ is compact. Let $\left(x_{k}\right) \subseteq \Omega_{0}$ be a minimizing sequence. That means $S\left(x_{k}\right) \rightarrow \gamma$ as $k \rightarrow \infty$. Thus, $T_{v_{1}}\left(x_{k} ; \Omega_{1}\right)<\gamma+1$ for sufficiently large $k$. For $t_{k}:=T_{v_{1}}\left(x_{k} ; \Omega_{1}\right)$, one has

$$
x_{k}+t_{k} v_{1} \in \Omega_{1} .
$$

Since $\Omega_{1}$ is compact, it is clear that $\left(x_{k}\right)$ has a convergent subsequence (without relabeling) to $\bar{x} \in \Omega_{0}$. Since $S$ is lower semicontinuous,

$$
S(\bar{x}) \leq \liminf _{k \rightarrow \infty} S\left(x_{k}\right)=\gamma
$$

Therefore, $\bar{x}$ is an optimal solution of the problem.

In the case where (2) is satisfied, we use a similar argument using the observation that $S$ is weakly lower semicontinuous since it is convex and lower semicontinuous. Moreover, every closed bounded convex set in a reflexive Banach space is weakly sequentially compact.

Theorem 5.2 Suppose that $\Omega_{0}$ is convex and that $\Omega_{i}$ are strictly convex for $i=1, \ldots, n$, $n \geq 2$. Suppose that any interval $[x, y], x, y \in \Omega_{0}, x \neq y$, does not intersect at least two sets among $\Omega_{i}$ for $i=1, \ldots, n$. Suppose further that any set of two vectors $\left\{v_{i}, v_{j}\right\}, i \neq j$, is linearly independent. Then the optimization problem (25) has at most one optimal solution.

Proof: We will show that $S$ is strictly convex on $\Omega_{0} \cap \operatorname{dom} S$. Suppose by contradiction that there exist $a, b \in \Omega_{0} \cap \operatorname{dom} S, a \neq b$, and $t \in(0,1)$ such that

$$
S(t a+(1-t) b)=t S(a)+(1-t) S(b) .
$$

This implies

$$
T_{v_{i}}(t a+(1-t) b)=t T_{v_{i}}(a)+(1-t) T_{v_{i}}(b) \text { for all } i=1, \ldots, n .
$$

Suppose $[a, b] \cap \Omega_{i}=\emptyset$ and $[a, b] \cap \Omega_{j}=\emptyset$ for $i \neq j$. Then $b-a \in \operatorname{span}\left\{v_{i}\right\} \cap \operatorname{span}\left\{v_{i}\right\}=\{0\}$, so $a=b$ by Proposition 2.7, which is a contradiction.

In what follows we are going to establish necessary and sufficient optimality condition for problem (25). For every $u \in X$, define

$$
I(u):=\left\{i \in\{1, \ldots, n\} \mid u \in \Omega_{i}\right\}
$$

and

$$
J(u):=\left\{i \in\{1, \ldots, n\} \mid u \notin \Omega_{i}\right\} .
$$

Theorem 5.3 Let $X$ be an Asplund space (see [7] for the definition). Consider the optimization problem (25). Suppose that $\bar{x} \in \Omega_{0}$ is an optimal solution of the problem and $T_{v_{i}}\left(\cdot ; \Omega_{i}\right)$ is Lipschitz continuous around $\bar{x}$ for all $i=1, \ldots, n$. Then there exist $x_{i}^{*} \in X^{*}$ with the following properties:

(1) $x_{i}^{*} \in N\left(\tilde{x}_{i} ; \Omega_{i}\right)$, where $\tilde{x}_{i}:=\Pi\left(\bar{x} ; \Omega_{i}\right)$ for $i=1, \ldots, n$.

(2) $\left\langle x_{i}^{*},-v_{i}\right\rangle \leq 1$ for all $i \in I(\bar{x})$ and $\left\langle x_{j}^{*}, v_{j}\right\rangle=-1$ for all $j \in J(\bar{x})$.

(3) $-\sum_{i=1}^{n} x_{i}^{*} \in N\left(\bar{x} ; \Omega_{0}\right)$. 
Proof: It is clear that $\bar{x} \in \Omega_{0}$ is an optimal solution of the optimization problem (25) if and only if it is a solution of the following unconstrained optimization problem:

$$
\operatorname{minimize} S(x)+\delta\left(x ; \Omega_{0}\right), x \in X
$$

where $\delta\left(\cdot ; \Omega_{0}\right)$ is the indicator function associated with $\Omega_{0}$ given by

$$
\delta\left(x ; \Omega_{0}\right):= \begin{cases}0 & \text { if } x \in \Omega_{0} \\ \infty & \text { otherwise. }\end{cases}
$$

Since each function $T_{v_{i}}\left(\cdot ; \Omega_{i}\right)$ is Lipschitz continuous at $\bar{x}$, using the limiting subdifferential sum rule from [7, Theorem 3.36], one has

$$
0 \in \partial\left[S(\cdot)+\delta\left(\cdot ; \Omega_{0}\right)\right](\bar{x})=\sum_{i=1}^{n} \partial T_{v_{i}}\left(\bar{x} ; \Omega_{i}\right)+N\left(\bar{x} ; \Omega_{0}\right)
$$

Then there exist $x_{i}^{*} \in \partial T_{v_{i}}\left(\bar{x} ; \Omega_{i}\right)$ such that

$$
-\sum_{i=1}^{n} x_{i}^{*} \in N\left(\bar{x} ; \Omega_{0}\right) .
$$

Finally, the $x_{i}^{*}$ for $i=1, \ldots, n$ satisfy (1) and (2) by Theorems 3.18 and 3.19 .

In the convex case, we are able to obtain necessary and sufficient optimality conditions under less restrictive assumption as in the theorem below.

Theorem 5.4 Let $X$ be a normed linear space and let $\Omega_{i}$ for $i=1, \ldots, n$ and $\Omega_{0}$ be convex sets. Consider the optimization problem (25). Suppose that there exist an element $u \in \operatorname{dom} S \cap \Omega_{0}$ at which all functions $T_{v_{i}}\left(\cdot ; \Omega_{i}\right)$ are continuous. If $\bar{x}$ is an optimal solution of the problem, then there exist $x_{i}^{*} \in X^{*}$ with the following properties:

(1) $x_{i}^{*} \in N\left(\tilde{x}_{i} ; \Omega_{i}\right)$, where $\tilde{x}_{i}:=\Pi\left(\bar{x} ; \Omega_{i}\right)$ for $i=1, \ldots, n$.

(2) $\left\langle x_{i}^{*},-v_{i}\right\rangle \leq 1$ for all $i \in I(\bar{x})$ and $\left\langle x_{i}^{*}, v_{j}\right\rangle=-1$ for all $j \in J(\bar{x})$.

(3) $-\sum_{i=1}^{n} x_{i}^{*} \in N\left(\bar{x} ; \Omega_{0}\right)$. Moreover, if $\bar{x} \in \Omega_{0}$ satisfies (1), (2), and (3), then $\bar{x}$ is an optimal solution of the problem.

Proof: Since $S$ is a convex function and $\Omega_{0}$ is a convex set, following the proof of Theorem 5.3 , one sees that $\bar{x} \in \Omega_{0}$ is an optimal solution of the optimization problem (25) if and only if

$$
0 \in \partial\left[S(\cdot)+\delta\left(\cdot ; \Omega_{0}\right)\right](\bar{x})=\sum_{i=1}^{n} \partial T_{v_{i}}\left(\bar{x} ; \Omega_{i}\right)+N\left(\bar{x} ; \Omega_{0}\right) .
$$

Then we use the well-known convex subdifferential sum rule and Theorem 3.2 to complete the proof.

Remark 5.5 (1) With the available subdifferential formulas for directional minimal time functions in the convex case from the paper, we are able to develop a numerical algorithm of subgradient type to solve problem (25) when the sets and the directions involved are of 
particular shapes. See [2] for more details on the theory of the subgradient method.

(2) Similar methods can be applied to the generalized Sylvester smallest enclosing ball problem stated as follows: given a finite number of nonempty closed target sets $\Omega_{i}$ for $i=1, \ldots, n$ and $n$ nonzero vectors $v_{i}$ for $i=1, \ldots, n$, and a nonempty closed constraint set $\Omega_{0}$, find a point $\bar{x} \in \Omega_{0}$ to place the initial points of the vectors such that the vectors can reach all the targets in the shortest time. This problem can be modeled as follows:

$$
\operatorname{minimize} \max \left\{T_{v_{i}}\left(x ; \Omega_{i}\right) \mid i=1, \ldots, n\right\} \text { subject to } x \in \Omega_{0} \text {. }
$$

\section{References}

[1] Aubin, J.-P, Frankowska, H.: Set-Valued Analysis. Birkhäuser, Boston (1990).

[2] D. Bertsekas, A. Nedic, and Ozdaglar, A.: Convex Analysis and Optimization, Athena Scientific, Boston, (2003).

[3] Clarke, F.H.: Optimization and Nonsmooth Analysis. Wiley, New York (1983).

[4] Clarke, F. H., Ledyaev, Yu. S., Stern, R. J., Wolenski, P. R.: Nonsmooth analysis and control theory. Graduate Texts in Mathematics, 178. Springer-Verlag, New York (1998).

[5] Colombo, G., Wolenski, P.R.: The subgradient formula for the minimal time function in the case of constant dynamics in Hilbert space. J. Global Optim. 28, 269-282 (2004).

[6] Gerstewitz (Tammer), Chr., Iwanow, E.: Dualität für nichtkonvexe vektorptimierungsprobleme, Wissenschaftliche Zeitschrift der Technischen Hochschule Ilmenau, 31, 61-81 (1985).

[7] Mordukhovich, B.S.: Variational Analysis and Generalized Differentiation, I: Basic Theory, II: Applications, Grundlehren Series (Fundamental Principles of Mathematical Sciences), Vols. 330 and 331, Springer, Berlin (2006).

[8] Mordukhovich, B.S., Nam, N.M.: Subgradients of minimal time functions under minimal assumptions. J. Convex Anal. 18, 915-947 (2011).

[9] Mordukhovich, B.S., Nam, N.M.: Applications of variational analysis to a generalized Fermat-Torricelli problem. J. Optim. Theory Appl. 148, 431-454 (2011).

[10] Rockafellar, R. T., Wets, R. J.-B.: Variational Analysis. Springer, Berlin (1998).

[11] Tammer, Chr., Zălinescu, C.: Lipschitz properties of the scalarization function and applications, Optimization 59, 305-319 (2010).

[12] Zălinescu, C.: Convex analysis in general vector spaces, World Scienctific, Singapore (2002). 